\title{
Cranial ultrasound for beginners
}

\author{
Pablo Caro-Domínguez ${ }^{1 \wedge}$, Catalina Lecacheux ${ }^{2,3}$, Cristina Hernandez-Herrera ${ }^{1}$, \\ Roberto Llorens-Salvador ${ }^{4} \wedge$
}

${ }^{1}$ Unidad de Radiología Pediátrica, Servicio de Radiodiagnóstico, Hospital Universitario Virgen del Rocío, Sevilla, Spain; ${ }^{2}$ Department of Radiology, University of Toronto, Toronto, ON, Canada; ${ }^{3}$ Department of Diagnostic Imaging, The Hospital for Sick Children, Toronto, ON, Canada; ${ }^{4}$ Unidad de Radiología Pediátrica, Servicio de Radiodiagnóstico, Hospital Universitario y Politécnico La Fe, Valencia, Spain

Contributions: (I) Conception and design: P Caro-Domínguez; (II) Administrative support: P Caro-Domínguez, C Lecacheux; (III) Provision of study materials or patients: All authors; (IV) Collection and assembly of data: All authors; (V) Data analysis and interpretation: None; (VI) Manuscript writing: All authors; (VII) Final approval of manuscript: All authors.

Correspondence to: Dr. Pablo Caro-Domínguez. Unidad de Radiología Pediátrica, Servicio de Radiodiagnóstico, Hospital Universitario Virgen del Rocío, Avenida Manuel Siurot S/N, CP 41013, Sevilla, Spain. Email: pablocaro82@hotmail.com.

\begin{abstract}
Cranial ultrasound (CUS) is an extremely valuable tool to evaluate the brain during the first year of life, in experienced hands. It is the initial screening imaging tool to evaluate the infants' brain and complementary to the use of computed tomography (CT) and magnetic resonance imaging (MRI). It is an accessible, inexpensive and harmless technique that can be used bedside as frequently as needed. The aim of this article is to provide a guide for beginners about the indications, basic technical parameters, scanning technique, standardized planes, common variants and the most frequent abnormal findings visualized with this technique. This article will explain a systematic technique to adequately visualize and document all the relevant intracranial structures, using the anterior fontanelle, mastoid fontanelle and Doppler ultrasound. The variants described in this review include the normal sulcation of the premature baby, the peritrigonal echogenic "blush", lenticulostriate vasculopathy (LSV), benign enlargement of the subrarachnoid space in infancy, asymmetric lateral ventricles, connatal cysts, cavum septum pellucidum (CSP), cavum vergae $(\mathrm{CV})$, cavum velum interpositum, megacisterna magna and choroid plexus cysts. This article will describe the sonographic appearance of different types of intracranial hemorrhage of the preterm and term baby, periventricular leukomalacia (PVL), central and peripheral hypoxic-ischemic events of the term baby, neonatal arterial infarction, cerebro-venous sinus thrombosis, congenital and neonatal cerebral infections, hydrocephalus, intracranial solid and cystic masses, and congenital brain malformations.
\end{abstract}

Keywords: Cranial; ultrasound; sonography; infant; neonate

Submitted Nov 22, 2020. Accepted for publication Jan 22, 2021.

doi: $10.21037 /$ tp-20-399

View this article at: http://dx.doi.org/10.21037/tp-20-399

\section{Introduction}

Cranial ultrasound (CUS) is an extremely valuable tool for the evaluation of the brain during the first year of life. It is used to look for haemorrhage or a hypoxic-ischemic event in the neonate, to exclude and monitor hydrocephalus, brain malformations, etc. (1). CUS has many advantages: it is safe (lack of ionizing radiation, no need for sedation), inexpensive, portable and can be quickly performed, and repeated as many times as necessary.

Although beginners in CUS might feel uncomfortable due to their lack of practice and familiarity with the imaging appearance of the diseases typical of this age group, using a

^ ORCID: Pablo Caro-Dominguez, 0000-0003-3540-6580; Roberto Llorens-Salvador, 0000-0002-2451-6699. 
systematic technique can help to confidently demonstrate the normal anatomy, characterize normal variants and identify pathologic findings, and more importantly not to miss relevant findings. The aim of this article is to introduce this technique to beginners, explain how to obtain a standardof-care CUS and to illustrate the normal appearance of the brain, normal variants and the most common pathologies.

\section{Indications of CUS in neonates and infants}

The most recent consensus in 2020 by the American Institute of Ultrasound in Medicine (AIUM) includes the following entities as indications for CUS in infants (1):

* Abnormal increase in head circumference;

* Haemorrhage or parenchymal abnormalities in preterm and term infants;

* Ventriculomegaly/hydrocephalus;

* Vascular abnormalities;

* Suspected hypoxic ischemic injury;

* Patients on hypothermia, extracorporeal membrane oxygenation (ECMO), and other support machines;

* Congenital malformations;

* Signs or symptoms of a central nervous system disorder (e.g., seizures, facial malformations, macrocephaly, microcephaly, and intrauterine growth restriction);

* Congenital or acquired brain infection;

* Suspected or known head trauma;

* Craniosynostosis;

* Follow-up or surveillance of previously documented abnormalities, including prenatal abnormalities;

* Screening before surgery.

\section{Cus technique}

In order to obtain a good set of images, the operator should know some basic factors of the ultrasound machine (2-4).

\section{Probe/transducer}

The choice of the proper transducer is extremely important to obtain the maximum information from the study. The use of at least two types of transducers, convex and linear, is recommended on every CUS. The convex to visualize deeper structures and the linear to characterize structures closer to the probe. Any area of abnormal echogenicity should be double checked with the other transducer, if possible, to confirm the finding.

In neonates, a small curvilinear transducer of approximately
$11 \mathrm{MHz}$ should be the first step while in a 6-month-old infant a curvilinear transducer of $8 \mathrm{MHz}$ will obtain better images due to the smaller size of the fontanelle and deeper location of the structures. The linear ultrasound (14-18 MHz) should be used in every patient to evaluate the superficial structures of the brain (cortex, extra-axial space, superior longitudinal sinus, etc.) and to evaluate deeper structures if possible, especially in preterm neonates.

\section{Parameters}

* Frequency: must be adapted to the structure evaluated and the age/size of the child. Seven point five to $10 \mathrm{MHz}$ are the adequate frequency for the initial scan of the entire brain, including the cerebrum, brainstem and cerebellum. Nine to $18 \mathrm{MHz}$ are utilized for a second more detailed evaluation of superficial anatomical structures.

* Depth: at the beginning of the study, the field of view of the scan should be adjusted to include the maximal depth of the cranium to visualize all cranial contents adequately.

* Gain: an adequate adjustment of gain helps not to miss important findings or to misdiagnose normal findings as pathology.

* Time-gain compensation curve (TGC): the TGC should be adjusted at the beginning of every study to avoid abnormal parenchymal 'brightness' in certain brain structures, which may be mistaken as brain oedema. Most modern ultrasound machines have a button to adjust it automatically.

* Focus: the focus should be moved throughout the study adapted to the region of interest. For the initial scan the focus should be positioned in the posterior fossa and moved depending on the findings.

\section{Standard planes/normal appearance of the brain}

A well-documented normal ultrasound of the brain should have no less than 20 images $(2,3)$; six images in the coronal plane with the curvilinear probe through the anterior fontanelle, five in the sagittal plane through the anterior fontanelle and two of the posterior fossa through the mastoid fontanelle. With the superficial linear transducer, the midline and parasagittal extra-axial space and cortex should be evaluated in the coronal and sagittal plane. Although in the premature infant due to the small size of the head and wide fontanelles a complete set of high resolution images of the brain can be obtained using 
a linear probe, similar to the convex probe protocol. Doppler evaluation of the anterior cerebral artery and superior sagittal sinus should also be performed in every ultrasound study (3).

The anterior fontanelle is the main window for scanning the infant brain (2-4). Scanning through the mastoid fontanelle should always be performed when the clinical situation of the patient allows it, because it helps to visualize pathologies of the posterior fossa (5). Other minor fontanelles (posterior and sphenoid) can be used if pathologies of these regions are suspected.

For an optimal study is essential to obtain a symmetric and centred image. Before putting gel on the patient's head (especially if they have lots of hair), the operator should find the fontanelle with the index finger and asses the size and location.

The baby can be positioned with the head parallel to the bed, or perpendicular, with the head closer to the ultrasound machine, depending on the preferences of the operator and clinical situation.

The operator should always be gentle with the baby's head and use as much warm gel as possible to have optimal acoustic transmission without air artefacts between the probe and the skin, avoiding compression. Once the operator identifies the best location in the fontanelle to visualize the intracranial contents, the transducer should not be moved, only tilted from anterior to posterior and right to left.

\section{Standard coronal planes of the brain through the anterior fontanelle (Figure 1)}

There are six standard images to achieve during the initial coronal scan of the anterior fontanelle (4):

(I) First coronal image demonstrates the frontal lobes, with the bony roof of the orbits inferiorly as the anatomical landmark (Figure 1A). This image should show symmetry of the frontal lobes, with the interhemispheric fissure in the midline. Grey matter shows low echogenicity and white matter homogeneous high echogenicity.

(II) Second coronal image includes the frontal horns of the lateral ventricles, frontal lobes, cavum septum pellucidum (CSP) and rostrum of the corpus callosum (Figure 1B). The size of the frontal horns can be symmetric or mildly asymmetric, that tend to normalize during the first year of life. The frontal horns of the lateral ventricles can be small within the first 24 hours of life, increasing slightly in size after that.
(III) Third coronal image is at the level of the third ventricle and includes the body of the lateral ventricles, the basal ganglia, thalami and Sylvian fissure (Figure 1C). The echogenicity and morphology of the cranial contents should be symmetric. However, symmetry does not exclude pathology. The Sylvian fissures may be wider in premature babies. Special attention should be paid to the caudo-thalamic groove in this view to exclude germinal matrix (GM) haemorrhage in preterm babies.

(IV) Fourth coronal image is at the level of the cerebellum (Figure 1D). It depicts the frontal and temporal lobes, deep grey nuclei, tentorium, mesencephalon, vermis and cerebellar hemispheres.

(V) Fifth coronal view is at the level of the trigones of the lateral ventricles with the choroid plexuses within them (Figure 1E). The cortex, subcortical, deep and periventricular white matter of the parietal and temporal lobes are included. The white matter shows high homogeneous echogenicity, less hyperechoic than the choroid plexus.

(VI) Sixth coronal image is at the level of the occipital lobes, demonstrating the low echogenicity of the cortex, higher echogenicity of the white matter and the interhemispheric fissure at the midline (Figure 1F).

\section{Standard sagittal planes of the brain through the anterior fontanelle (Figure 2)}

A minimum of five standard sagittal images should be obtained by tilting the transducer from the midline to lateral, first to the right and secondly to the left (4):

(I) Midline sagittal view (Figure 2A): this image depicts the midline anatomical structures; the corpus callosum, the midline view of the entire ventricular system, the midbrain and cerebellar vermis.

(II) Parasagittal right and left views (Figure 2B): these images show the lateral ventricle with a "C-shape", thalami, basal ganglia and periventricular white matter. On this image, the caudo-thalamic groove should be seen separating the caudate nucleus anteriorly and the thalamus posteriorly.

(III) Lateral parasagittal right and left views (Figure 2C): these are obtained laterally at the level of the Sylvian fissure, including the white matter and cortex of the frontal, parietal and temporal lobes. 

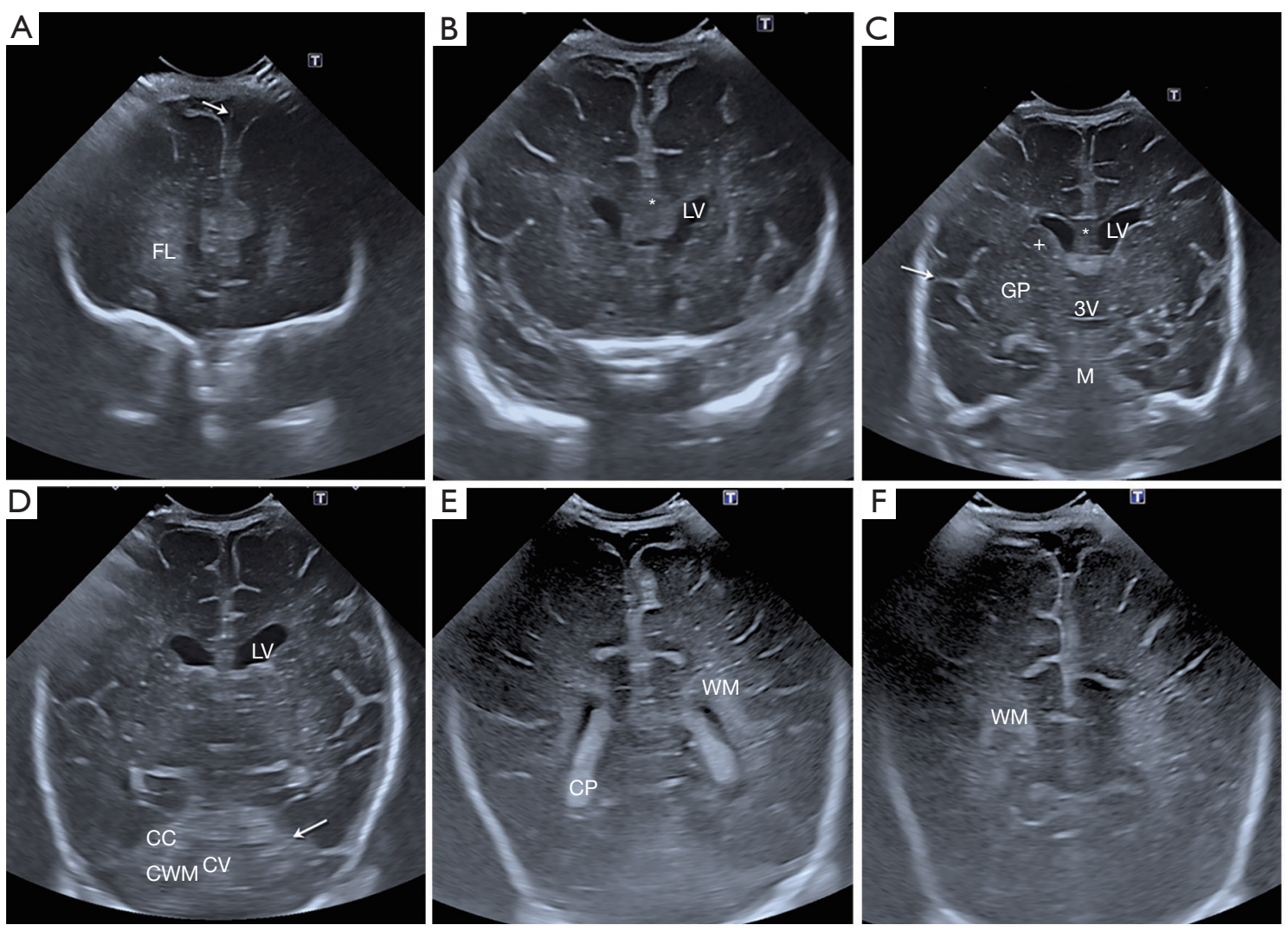

Figure 1 Fourteen-day-old baby girl with intrauterine growth restriction (images obtained with an 11-MHz probe). (A) First standard coronal image through the frontal lobes (FL) with an arrow pointing the interhemispheric fissure. (B) Second coronal image through the frontal horns of lateral ventricles (LV), with an asterisk in the corpus callosum. (C) Third coronal section through the third ventricle (3V), including the LV, caudate nucleus (+), globus pallidus (GP), midbrain (M), corpus callosum (asterisk) and Sylvian fissures (arrow). (D) Fourth coronal section at the level of cerebellum, including the vermis (CV), cerebellar white matter (CWM), cerebellar cortex (CC) and tentorium (arrow). (E) Fifth coronal section through the ventricular trigone, delineating the choroid plexus (CP), within the LV and the periatrial white matter (WM). (F) Sixth coronal section through the occipital lobes, including the posterior periventricular WM.
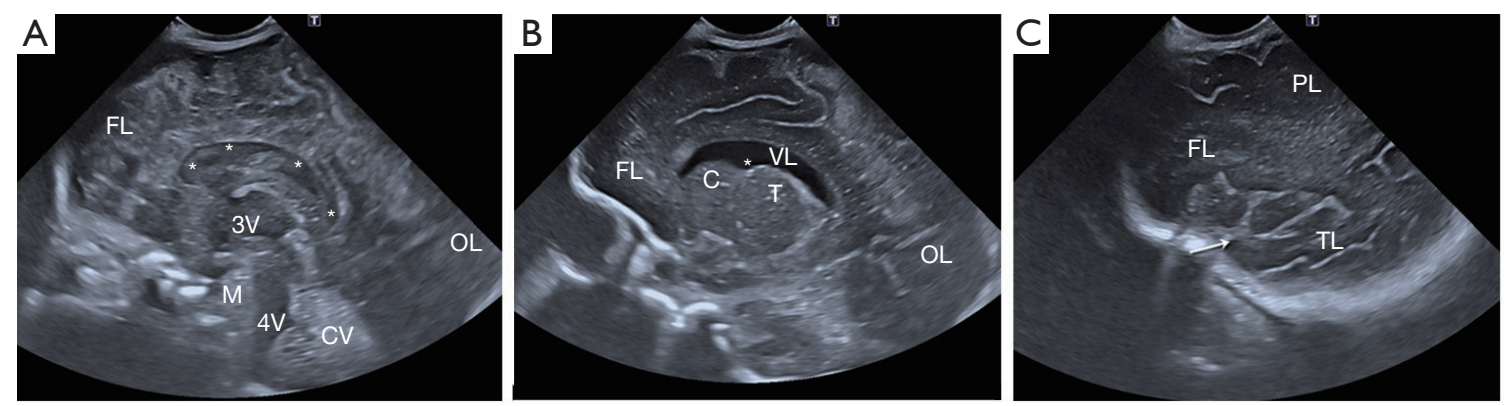

Figure 2 Same patient of Figure 1. (A) Midline sagittal image including the entire corpus callosum (asterisks), the entire midline ventricular system, including the third $(3 \mathrm{~V})$ and four ventricles $(4 \mathrm{~V})$, the cerebrum from the frontal (FL) to the occipital lobes (OL), the midbrain (M) and cerebellar vermis (CV). (B) A parasagittal image shows the caudo-thalamic groove (asterisk), with the caudate nucleus (C) and thalamus posteriorly (T). (C) A tangential parasagittal section demonstrated the frontal (FL), parietal (PL) and temporal (TL) lobes and the Sylvian fissure (arrow). 

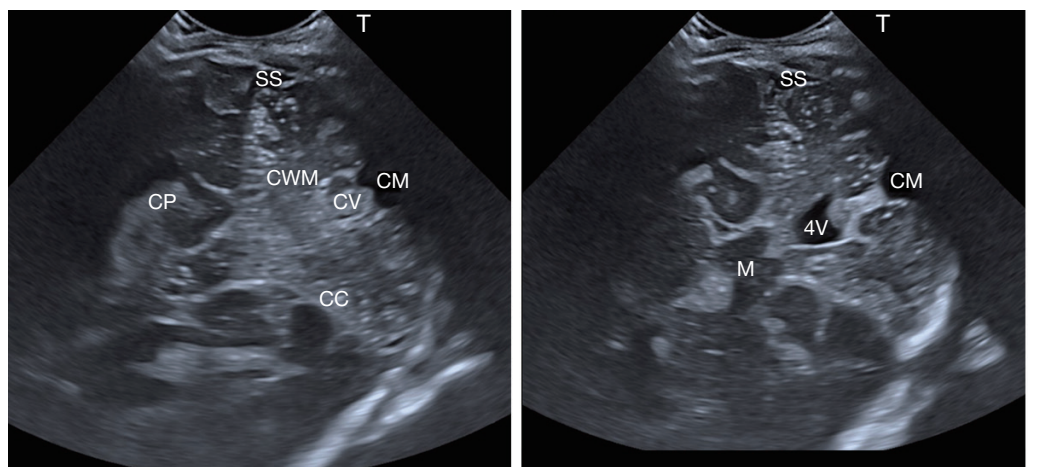

Figure 3 Coronal images of the posterior fossa obtained through the mastoid fontanelle of the same patient. The cerebellar cortex (CC), white matter (CWM), vermis (CV), sigmoid sinus (SS), fourth ventricle (4V) and cisterna magna (CM) are identified in the posterior fossa. The temporal lobe and the temporal horn of the lateral ventricles, with the choroid plexus $(\mathrm{CP})$ within it is also seen above the tentorium.

\section{Coronal view of the posterior fossa through the mastoid fontanelle (Figure 3)}

This section is obtained by placing the transducer behind the patient's ear, coronal to the posterior fossa pointing towards the cerebellum and tilting from anterior to posterior (5). These images show the midbrain, fourth ventricle, cerebellar vermis, both cerebellar hemispheres, the sigmoid sinus, tentorium and the cisterna magna. The caudal aspect of the temporal lobe and the temporal horn of the lateral ventricles can also be seen through this fontanelle.

\section{Doppler ultrasound: color and pulsed Doppler}

Doppler ultrasound is valuable to study cerebral hemodynamics and should be used to assess the intracranial vasculature, because many cerebral lesions in newborns and infants are of vascular origin. Colour and power Doppler techniques are indicated to explore cerebral vessels and confirm their normal blood flow $(2,3)$. For instance, direct interrogation of the dural venous sinuses through the anterior, posterior and mastoid fontanelles is helpful to exclude thrombosis (6). Pulsated Doppler of the anterior cerebral or pericallosal arteries must be performed in every patient to assess for spectral analysis. Abnormal curves and resistive index values are important in entities like neonatal hypoxic-ischaemic encephalopathy (HIE) (3).

\section{Normal variants of the brain ultrasound appearance}

The beginner on CUS should be familiar with some normal variants, typical of this age group.

(I) Normal sulcation of the premature baby. The development of the brain in the fetus is a very complex and progressive process throughout pregnancy and after birth (7). The surface of the brain is smooth in premature babies, with progressive appearance of new sulci and gyri (Figure 4). At term, the number and appearance of sulci and gyri should be similar to a teenager. Preterm babies under 28 weeks have very few sulci, therefore the lack of sulcation in extreme prematures should not be confused with an extensive cortical malformation (7). On the contrary, a smooth surface of the brain, with only a few sulci in a term baby should suggest the diagnosis of an extensive cortical malformation. In any case, if a sulcation anomaly is suspected, comparison with other children of the same age or an atlas is extremely useful.

(II) Peritrigonal echogenic "blush". It refers to an area of symmetric homogeneous high echogenicity of the periventricular white matter around the posterior horn of the lateral ventricles, parallel to the ventricular ependymal surface (Figure 5). It is frequently seen in preterm infants and disappears during the first month of life (4). The main differential diagnoses include cerebral haemorrhage and periventricular leukomalacia (PVL). The keys to differentiate them are that the echogenic "blush" is symmetric, homogeneous and less echogenic than the choroid plexus.

(III) Lenticulostriate vasculopathy (LSV). They are 

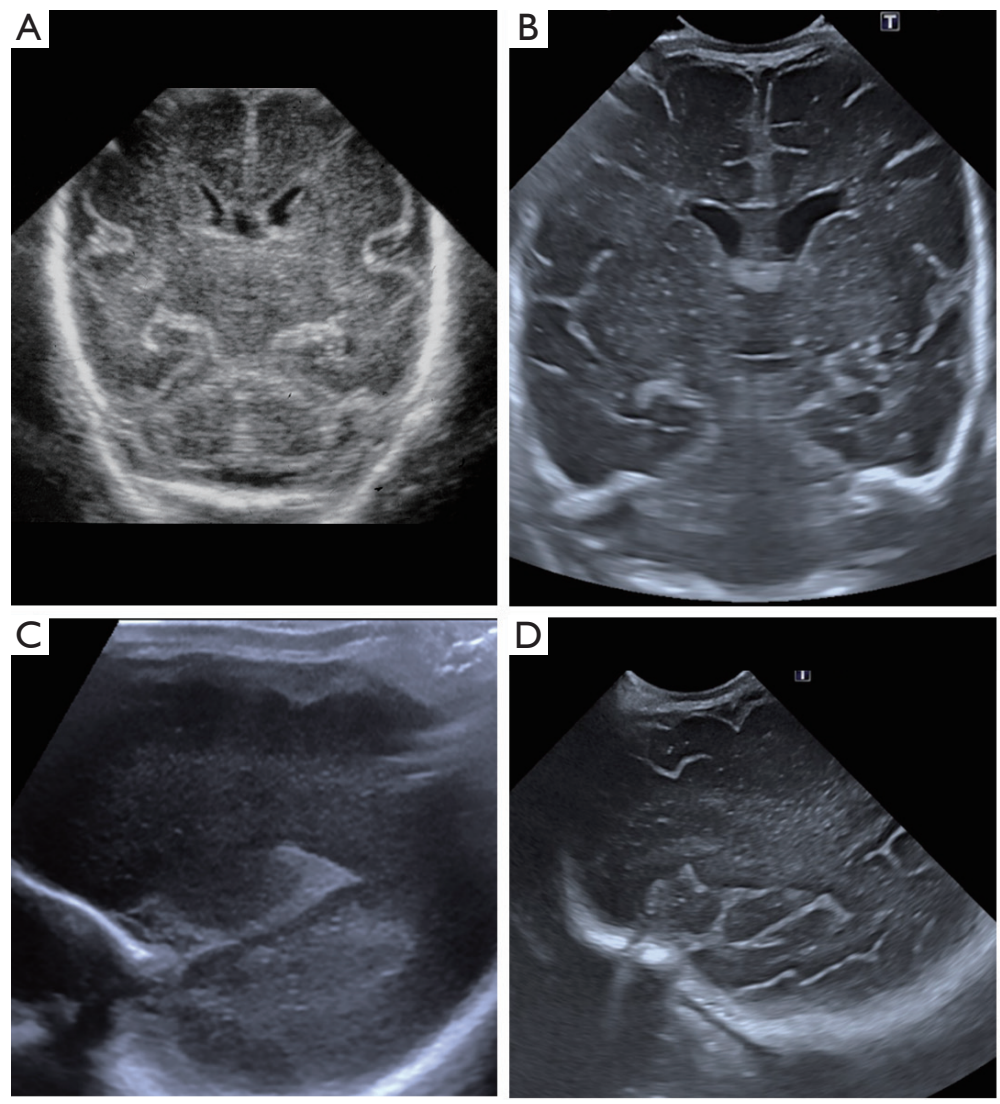

Figure $4(\mathrm{~A}, \mathrm{~B})$ are coronal ultrasound images of preterm and term babies respectively where the progressive formation of the sulcation and gyration during gestation can be identified. The same progression can be seen in (C,D) on the sagittal plane of a preterm and a full-term baby, respectively.

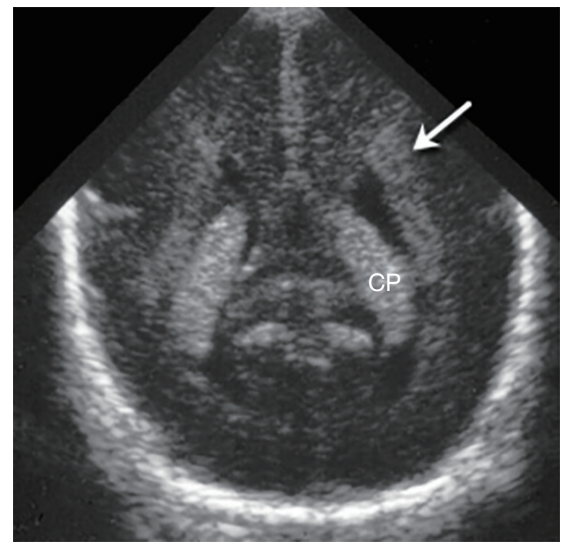

Figure 5 Coronal ultrasound image of a 29-week-old preterm, illustrating the peritrigonal "blush" (arrow). Note that the high signal of the periventricular white matter is homogeneous, relatively symmetric and less hyperechogenic than the choroid plexus (CP). linear foci of high echogenicity with a branchingpattern in the region of the basal ganglia, that can be bilateral or unilateral. It is a non-specific finding (Figure 6) which was associated in the past with some pathologies (4). Nowadays, it is divided in low grade (one or two fine striations) and high grade (with three striations or more) (8). Low grade LSV is considered a variant, probably seen more often due to the modern ultrasound machines with higher image resolution (Figure 6) (8). On the other hand, high-grade LSV can be associated with hypoxic-ischemic events and infections (8).

(IV) Benign enlargement of the subarachnoid space (BESS) in infancy. It is a symmetric widening of the frontal and parietal extra-axial space in infants (Figure 7), typically referred for macrocephaly. It usually occurs more in males than females, with a familial predilection and the condition resolves 

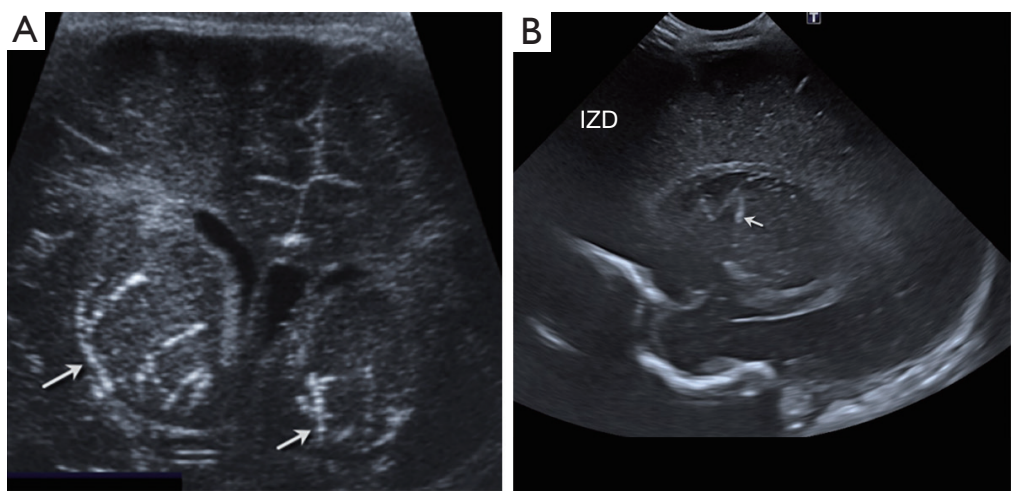

Figure 6 Coronal (A) and sagittal (B) ultrasound images obtained with an 11-MHz probe of a 31-week-old baby girl illustrating lenticulostriate vasculopathy (LSV) (arrows) as linear foci of high echogenicity with a branching pattern in the region of the basal ganglia.
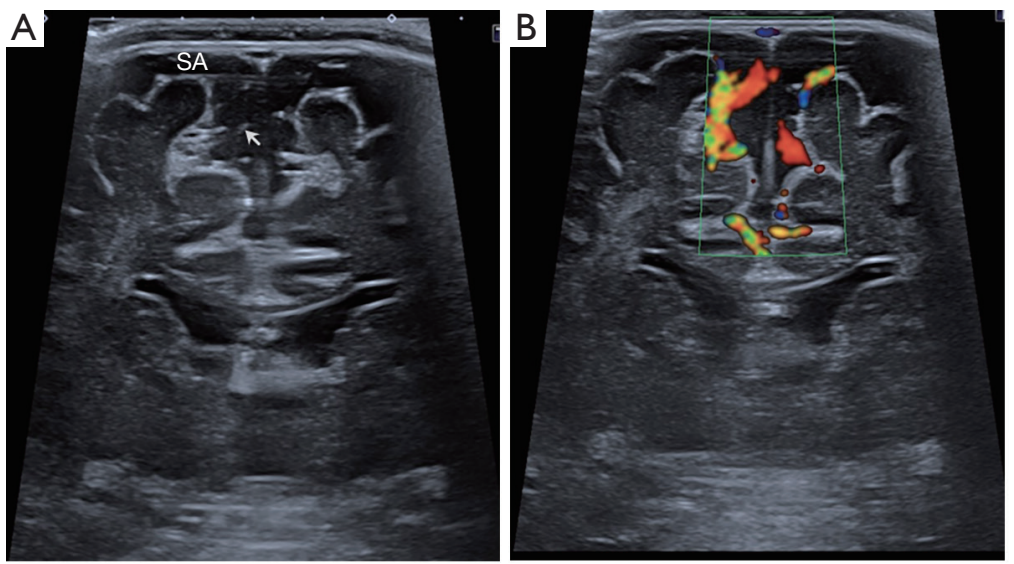

Figure 7 Coronal mode B (A) and colour Doppler (B) ultrasound images obtained with a linear 14-MHz probe of a 6-month-old baby boy illustrating the benign enlargement of the subarachnoid space (BESS) in infancy. There is bilateral symmetric widening of the frontal subarachnoid space (SA), with a width of $7 \mathrm{~mm}$. Note the linear foci of high echogenicity within the subarachnoid space, representing normal cortical veins (arrow), differentiating this condition from subdural fluid collections, confirmed with colour Doppler ultrasound.

spontaneously by the age of 2 years (4). In this variant, the width of the interhemispheric extraaxial space measures between 5 and 10 millimetres. There is no flattening of adjacent gyri, the CSF space follows the gyral contour and the adjacent cerebral cortex does not show abnormalities. A key distinction between BESS and a subdural fluid collection, is that in the former, cortical veins are identified in the subarachnoid space as hyperechogenic linear structures, while in a subdural collection, no vessel is identified within the extraaxial space. Colour Doppler is very useful to confirm the presence of subarachnoid cortical veins (Figure $7 B$ ).

(V) Asymmetric lateral ventricles. Mild asymmetry in the size of the lateral ventricles is a common finding in preterm newborns (Figure 8) that tends to normalize during the first month of life (4), but may persist in older age. It should not be associated with abnormal echogenicity or volume loss of the periventricular white matter.

(VI) Connatal cysts, also known as coarctation of the lateral ventricles or frontal horn cysts (Figure 9). They are small cystic lesions adjacent to the superolateral margins of the frontal horns of the lateral ventricles $(3,9)$. They are considered a normal variant and can be single or multiple, unilateral or bilateral and they tend to disappear within the first year of life. They should be distinguished from other cystic structures, such as GM cysts, 

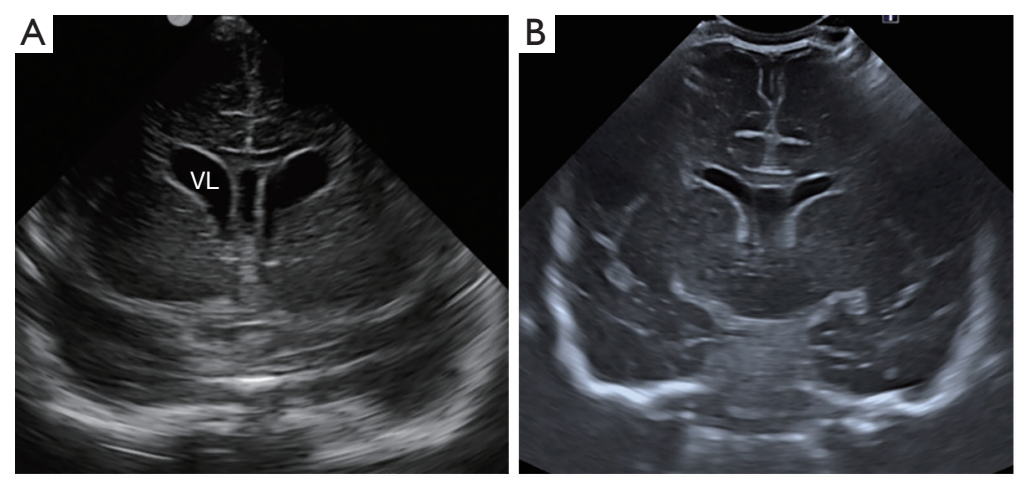

Figure 8 Coronal ultrasound images through the frontal horns in a 31-week-old preterm baby girl at birth (A) and 4 weeks (B) later illustrating the mild asymmetry of the lateral ventricles at birth (VL) with the left side more prominent which becomes progressively symmetric and also the progressive normalization of the mild ventricular distention of preterm babies. The cavum septum pellucidum (CSP) can also be identified in (A), between the frontal horns of the lateral ventricles.
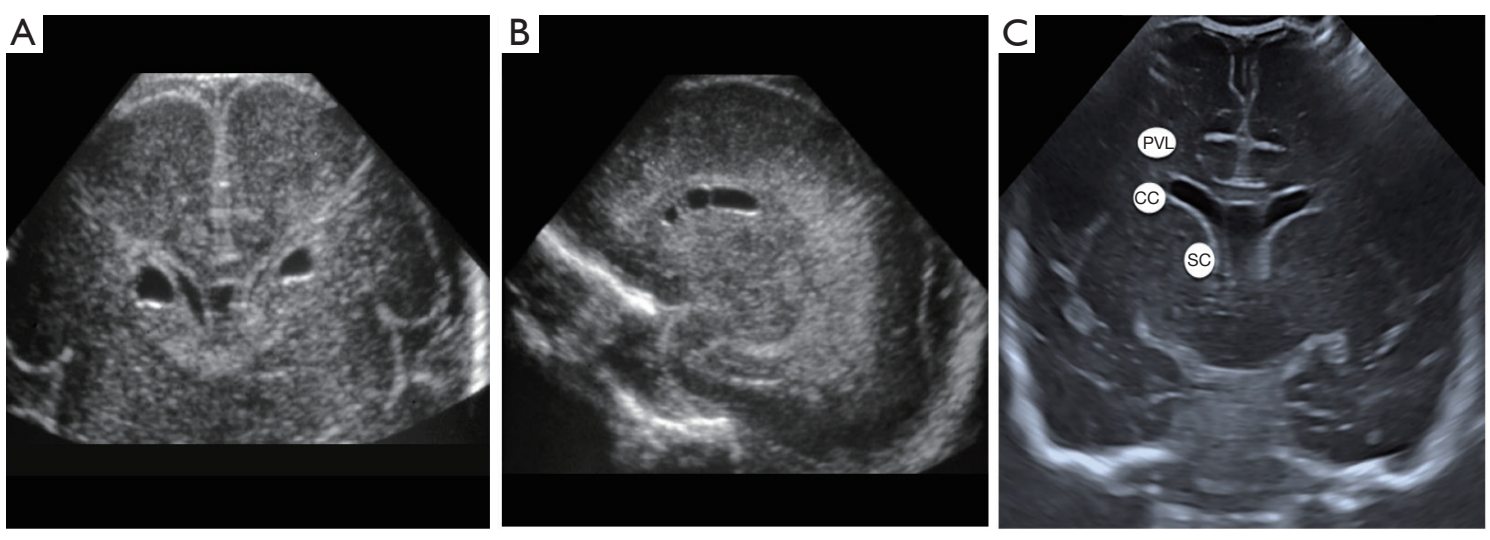

Figure 9 Coronal (A) and sagittal (B) ultrasound images of the frontal horns of the lateral ventricles, obtained with a 14-MHz linear probe, illustrating bilateral connatal cysts (CC), in a 27-week-old premature. (C) is a normal ultrasound image illustrating the location of CC, a normal variant, compared with two pathologic conditions, periventricular leukomalacia (PVL) and subependymal cysts (SC).

subependymal cysts or cystic PVL (9). As illustrated in Figure 9C, the location of the cysts in relation to the lateral surface of the lateral ventricles allows to differentiate these entities. PVL tends to be in the deep white matter superior to the lateral ventricle, GM cysts in the caudo-thalamic groove and subependymail cysts lateral to the groove. Connatal cysts are located in continuation to the external angle of the lateral ventricles.

(VII) CSP, cavum vergae (CV) and cavum veli interpositi (CVI) (Figure 10). CSP and CV is a midline fluid filled cavity located between the lateral ventricles. They are a single cavity, with two different names depending on its location: anterior to the foramen
Monro is called CSP and posterior to it, CV (3). The septum pellucidum is a marker of normal development of the cerebral midline structures. It begins to form around the third month of gestation and the closure starts approximately at 6 months of gestation and progresses from posterior to anterior. Most of the times, it has disappeared at birth. The CSP and CV can be normal in foetuses, preterm and term babies and it is considered a normal variant in older children and adults (9). Non-visualisation of the CSP within the 18 and 37 weeks of gestation is a prenatal sonographic finding, which in most cases is associated with neuroanatomical anomalies that include agenesis 

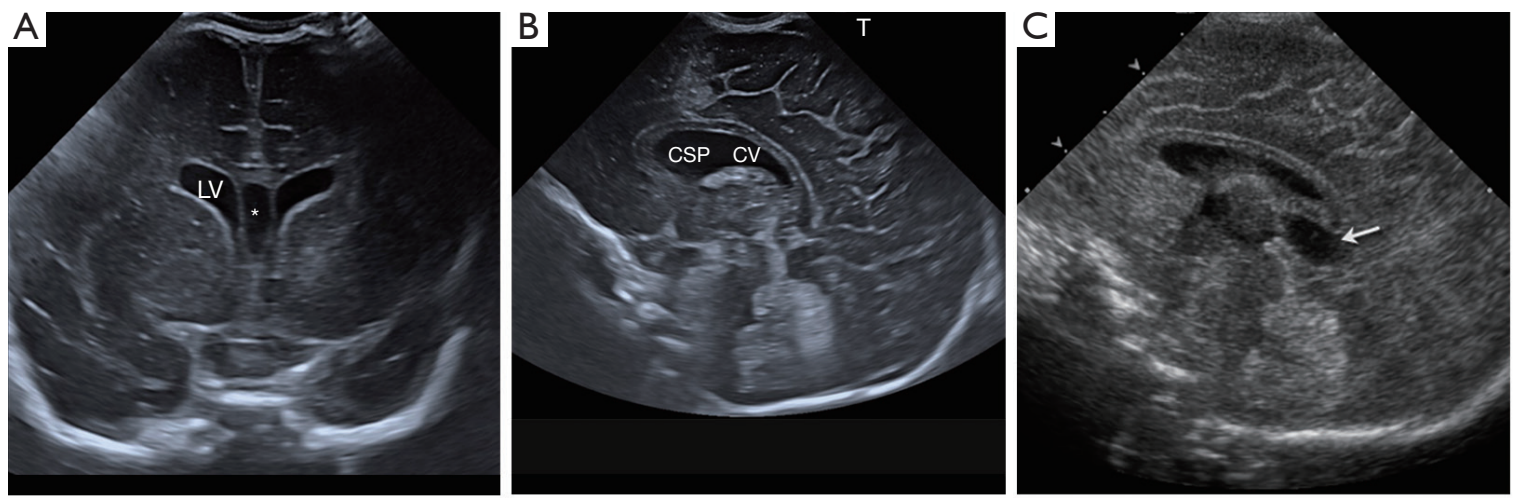

Figure 10 Coronal (A) and midsagittal (B) ultrasound images obtained with an $11-\mathrm{MHz}$ probe in a 1 -week-old preterm baby born at 30-week of gestation illustrating the presence of cavum septum pellucidum (CSP) and cavum vergae (CV) [asterisk in (A), CV in (B)]. (C) is a midsagittal ultrasound image of the brain of a term newborn showing the cavum velum interpositum (arrow).

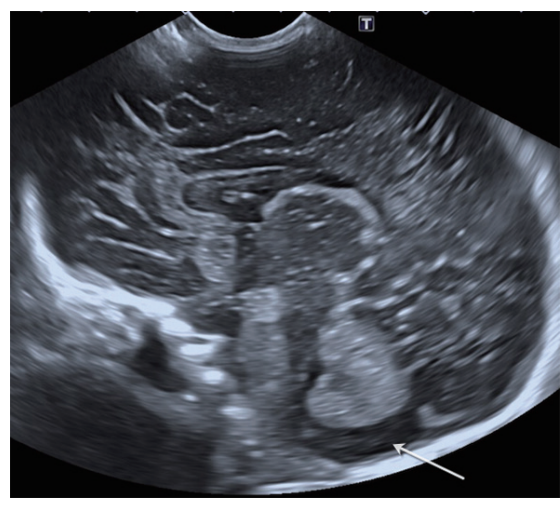

Figure 11 Midline sagittal ultrasound imagen obtained with an $11-\mathrm{MHz}$ transducer illustrating megacisterna magna (arrow) in a term baby boy. Note the absence of mass effect, normal size of the cerebellar vermis and posterior fossa.

of the corpus callosum, schizencephaly, septooptic dysplasia, holoprosencephaly, chronic hydrocephalus and acquired fetal brain injury (10). CVI is simple unilocular thin-walled cystic lesion in the pineal region, between the fornices of the corpus callosum and above the internal cerebral veins (4). It does not have mass effect (difference from a pineal arachnoid cyst), neither has internal blood flow (difference from the malformation of the vein of Galen).

(VIII) Megacisterna magna. It refers to focal accumulation of cerebrospinal fluid inferior and dorsal to the cerebellar vermis (Figure 11). It can measure up to $12 \mathrm{~mm}$ on antenatal imaging. It does not have mass effect, nor bone scalloping (difference from arachnoid cyst) and the size, location and morphology of the cerebellar vermis, tentorium and posterior fossa are within normal limits (difference from Blake's pouch cyst and Dandy Walker malformation) (4).

(IX) Choroid plexus cysts. A small cyst within the choroid plexus is a common incidental finding (Figure 12), without clinical significance, usually unilateral and not associated with genetic abnormalities (3).

\section{Pathologic findings}

CUS is an excellent imaging modality to evaluate brain abnormalities in neonates and infants and to follow-up their sequelae $(2,3)$. It has a complementary role with CT and MRI. Ultrasound can be used as the screening tool for most of the pathologies, CT is useful for emergencies, unstable patients and post-trauma. MRI is nowadays the goldstandard technique to evaluate the vast majority of cerebral pathologies due to its high soft-tissue anatomical resolution and lack of radiation. However, it is a longer study that may require general anesthesia or sedation.

\section{Haemorrbage}

\section{GM or intraventricular haemorrhage in the preterm infant (GM/IVH)}

The neurons form at the GM, located in the wall of the lateral ventricles, and migrates though the white matter to the cortex. The GM is avidly vascularized and susceptible to bleed in case of hemodynamic disturbances (7). 

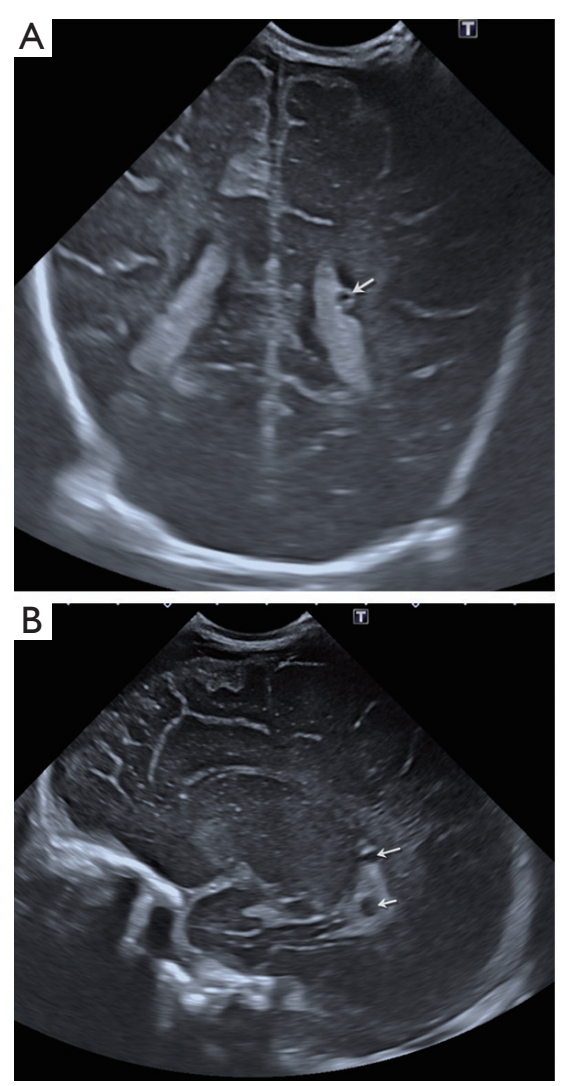

Figure 12 Coronal (A) and sagittal (B) ultrasound images obtained with a $14-\mathrm{MHz}$ probe of a 1 -month-old baby girl demonstrating two small simple cysts (arrows) in the left choroid plexus.

Intraventricular or GM haemorrhage of the premature occurs primarily in those at gestational ages of less than 30 weeks, with birth weight less than 1,500 g, or both (7). Most haemorrhages (90\%) occur in the first 7 days of life, but only one third occur in the first 24 hours. The optimal time for scanning is approximately 72 hours after birth (3). All children at risk should have an ultrasound by 7 to 10 days of age and repeat cranial sonographic screening at 4 to 6 weeks of age $(7,11)$.

GM/IVH is divided in four grades:

(I) Grade 1, or GM haemorrhage, originates within the GM located within the caudo-thalamic groove (Figure 13). It is contained by the ependyma and presents as a homogeneous, moderately to highly echogenic mass in the caudo-thalamic groove, when compared to the echogenicity of the adjacent choroid plexus. With time the hematoma retracts, haemorrhage resolves and a small cystic change may develop in this region before complete resolution.
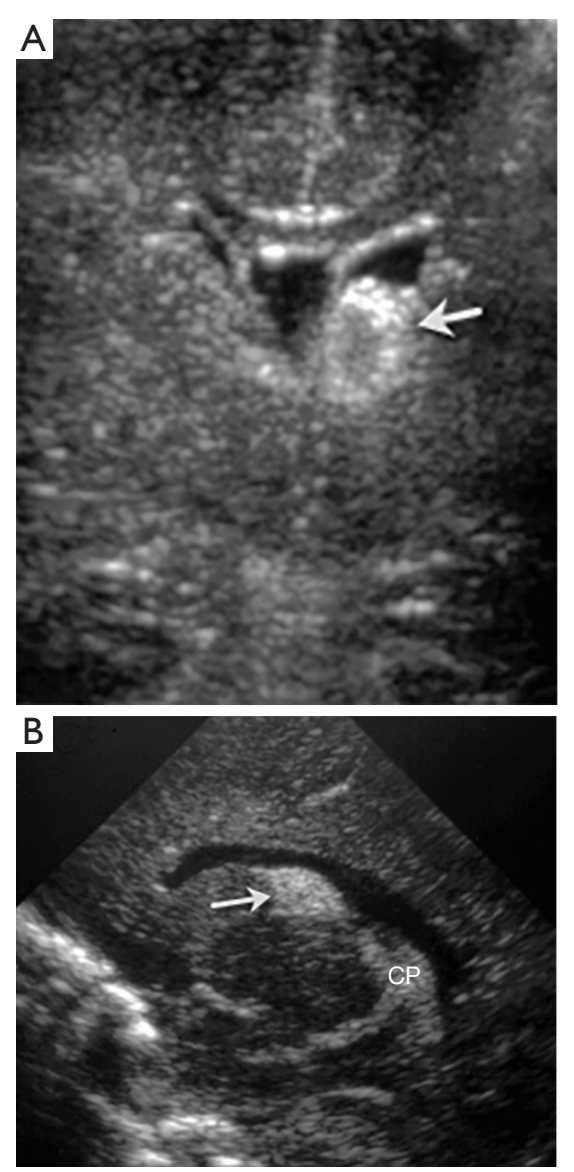

Figure 13 Twenty-nine-week-old baby with left germinal matrix (GM) hemorrhage. Coronal (A) and sagittal (B) ultrasound images show a focus of high echogenicity in the left caudo-thalamic groove (arrow) in keeping with GM hemorrhage. Note the choroid plexus posteriorly (CP) with similar echogenicity.

(II) IVH grade II develops when the clot opens into the ventricular system. It may appear as a thickened choroid plexus, compared to the contralateral one. Careful evaluation of the occipital horn is important if the bleeding is small and ventricles have a normal size, because blood tends to deposit in the dependent portion of the ventricle when the baby is laying down. The mastoid fontanelle view is important to depict blood within the 4th ventricle (4).

(III) IVH grade III or with ventricular dilation (Figure 14). Post-haemorrhagic ventricular dilation (PHVD) might develop due to obstruction of the foramina by blood products or by direct expansion of the ventricles by the clot (7). With the 


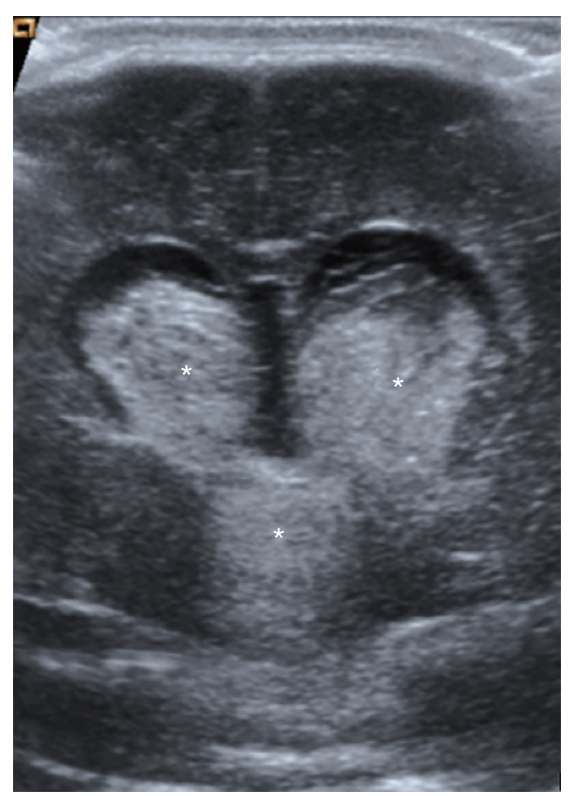

Figure 14 Coronal ultrasound image obtained with the curvilinear $11-\mathrm{MHz}$ probe that demonstrate bilateral intraventricular haemorrhage (IVH) (asterisks) in the frontal horns of the dilated lateral ventricles extending to the third ventricle.

development of PHVD, the clot and the choroid plexus becomes better defined. The blood in the CSF causes a chemical ventriculitis, with thickening and increased echogenicity of the subependymal lining of the ventricle.

(IV) Periventricular Ischaemic-Haemorrhagic Infarction (formerly known as grade IV) consists in parenchymal injury associated with a previous grade I to III GM/IVH (Figure 15). It appears as an echogenic fan-shaped mass in the brain parenchima adjacent to the site of IVH caused by venous infarction, secondary to a large hematoma compressing subependymal veins causing ischemia and haemorrhage (7). These periventricular white matter haemorrhagic infarcts follow a frontal to parieto-occipital orientation and are asymmetrical, usually unilateral. The intraparenchymal damage caused by the venous infarction, causes necrosis and porencephaly in this region 2 or 3 months after the event. These children may develop cerebral palsy (7).

\section{Parenchymal haemorrhage}

Parenchymal brain haemorrhage although less common may be seen in term neonates, secondary to cerebral
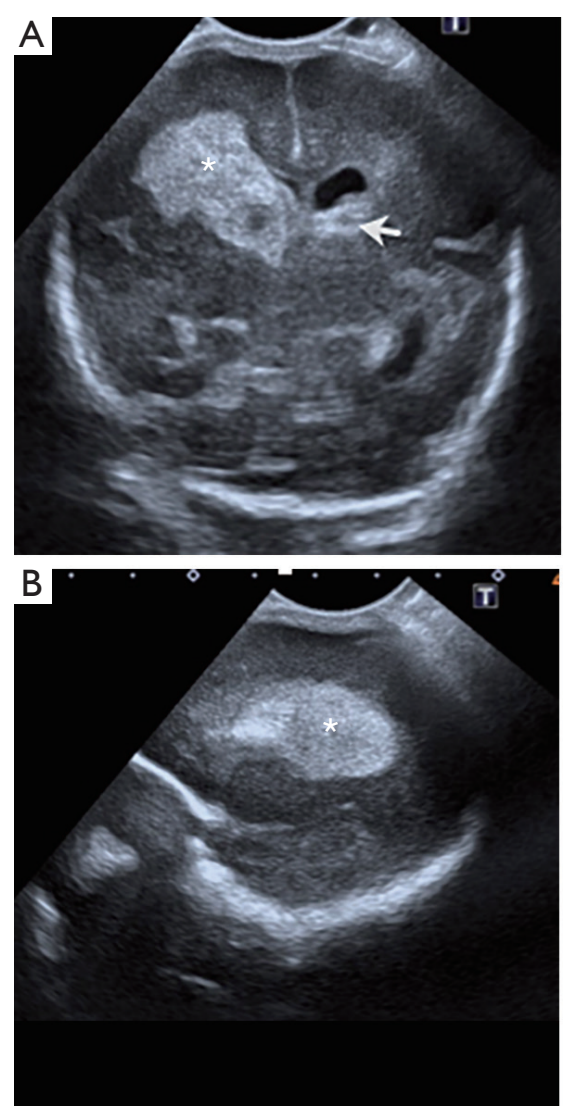

Figure 15 Twenty-eight-week-old baby boy with right parenchymal venous infarction (formerly known as grade 4). Coronal (A) and sagittal (B) ultrasound images obtained with a curvilinear 11- $\mathrm{MHz}$ probe show a large hyperechogenic focus of hemorrhage in the right caudothalamic groove that extends to the periventricular white matter (asterisk). Note a small focus of germinal matrix (GM) hemorrhage on the left side (arrow), with mild dilatation of the ventricle.

sinus thrombosis, coagulopathy, infection, heparinization, hypoglycemia, tumours, genetic conditions or vascular malformations (2). Choroid plexus haemorrhage is not common and tends to be more of a term event (3). ECMO is a risk factor for intracranial bleeding and needs to have daily ultrasound screening (3).

Cerebellar haemorrhage is also less common and frequently seen as a complication of a traumatic delivery in full-term infants, in ECMO therapy, with coagulopathy and in extremely preterm newborns $(3,12)$. It is important to use the mastoid fontanelle view to evaluate cerebellar haemorrhage that might be missed with the anterior fontanelle approach (Figure 16). However, small cerebellar haemorrhages $(<4 \mathrm{~mm})$ are missed with CUS using the 

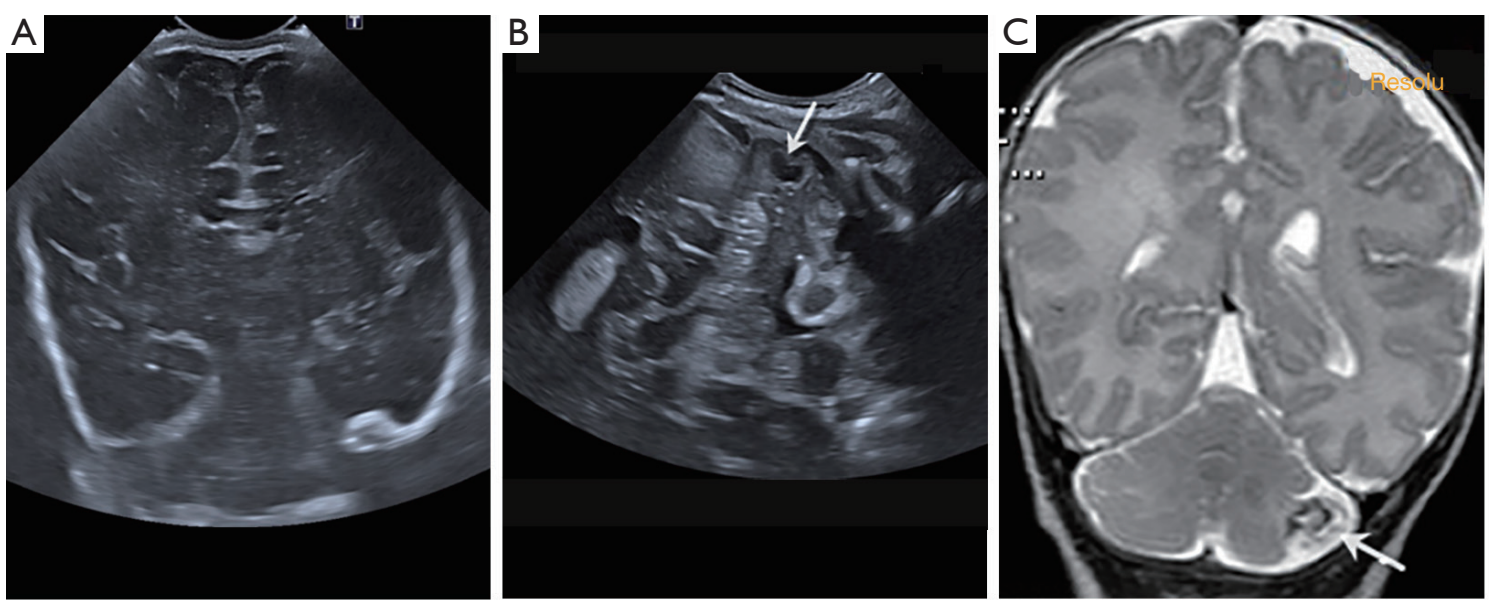

Figure 16 Twenty-seven-week-old baby girl (750 grams at birth). Coronal ultrasound image through the anterior fontanelle (A), performed at day 10, where is difficult to depict the abnormality. Coronal ultrasound image through the left mastoid fontanelle (B) delineates a round focus of low echogenicity in the left cerebellar hemisphere suggestive of an area of post-hemorrhagic porencephaly (arrow). A coronal magnetic resonance $\mathrm{T} 2$ image $(\mathrm{C})$ confirms the finding.
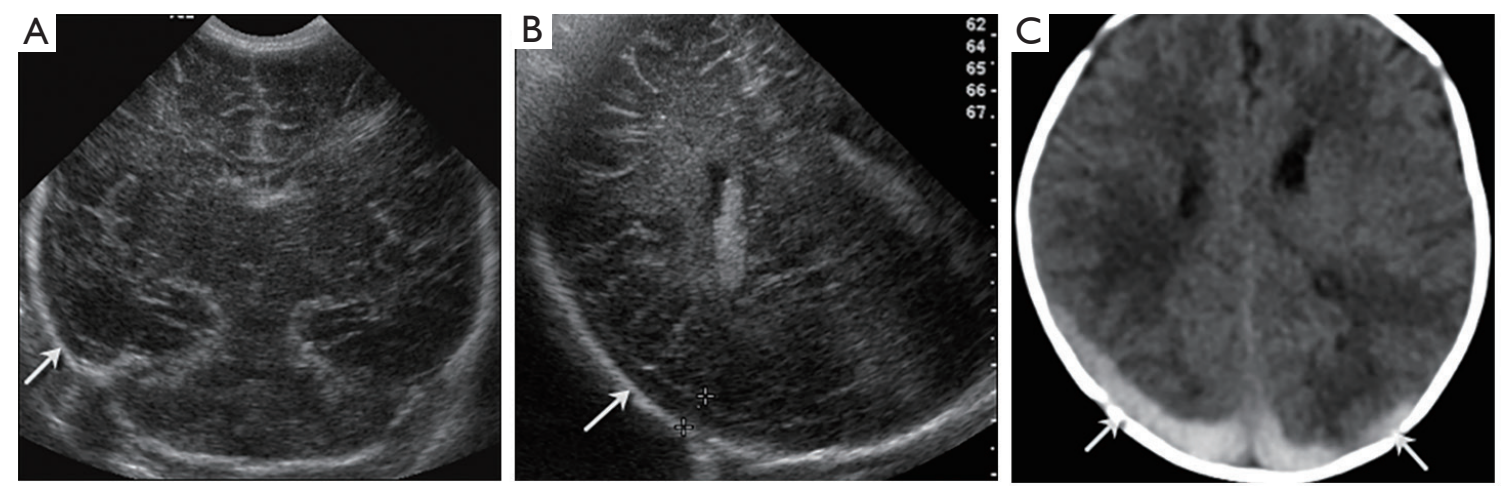

Figure 17 Neonate after meconium aspiration. Evaluation prior to extracorporeal membrane oxygenation (ECMO). Coronal ultrasound $(\mathrm{A}, \mathrm{B})$ images show a subdural collection encircling the right cerebral hemisphere (arrows). An axial computed tomography (CT) image (C) shows bilateral laminar subdural acute haemorrhages (arrows).

anterior and mastoid views (13).

\section{Extraaxial haemorrhage}

Subdural haemorrhage is not uncommon in newborns after a traumatic birth, without significant sequelae (Figure 17). It can also be seen in non-accidental injury, genetic and bleeding disorders (3).

Epidural haemorrhage can be seen in neonates who required forceps during delivery. If the baby is unstable or has decreasing levels of haemoglobin, CT is the preferred imaging modality to evaluate intracranial post-traumatic findings, especially fractures and extra-axial haematomas in the convexities.

Subarachnoid haemorrhage can be seen after asphyxia, trauma, or disseminated intravascular coagulation. CUS may help to delineate enlarged interhemispheric and sylvian fissures with thickened sulci (3). However, ultrasound is limited to investigate this finding and CT is the preferred imaging modality.

\section{Hypoxic-ischemic events}

The sonographic appearance of a hypoxic-ischemic event in the newborn brain depends on the prematurity and the 

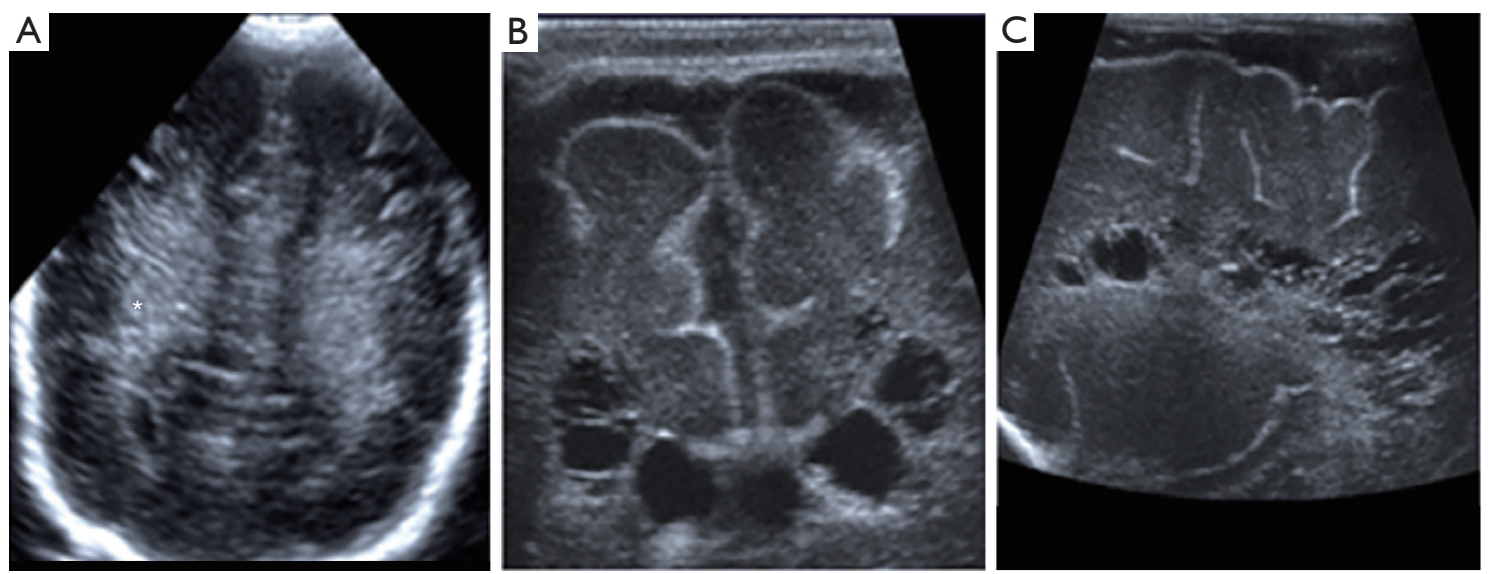

Figure 18 Twenty-seven-week-old preterm baby with perinatal asphyxia. Coronal (A) ultrasound obtained at day 7 demonstrate abnormal increased echogenicity of the periventricular white matter (star). Follow-up magnified coronal (B) and sagittal (C) ultrasound images obtained 3 weeks later demonstrated cystic periventricular leukomalacia (PVL) in the same areas.

length and severity of the event $(2,3,7,14)$.

\section{Premature white matter injury/PVL}

This condition associated with a hypoxic-ischemic event affects predominantly the premature infant, and it is closely related to cerebral palsy (7). It is the result of decreased oxygen delivery (hypoxia) and reduced cerebral perfusion (ischemia) for a metabolically very active neonatal brain. Risk factors include perinatal asphyxia, circulatory compromise, recurrent apneas, sepsis, maternal chorioamnionitis and severe respiratory distress syndrome (3).

It is characterized by infarction and necrosis of the white matter and sparing the grey matter/cortex (7). By CUS the initial appearance are patchy areas of heterogeneous increased echogenicity affecting the white matter, predominantly periventricular, adjacent to the frontal horns and the atrium of the lateral ventricles (Figure 18). These foci tend to be bilateral, slightly asymmetrical. Volume loss and cystic cavities develop in many cases a few weeks after the event. Thinning of the corpus callosum is associated to PVL, because of white matter necrosis and decreased number of crossing fibres. Follow up examinations should be done 4 weeks after birth to evaluate evolving PVL (7).

\section{HIE of the term neonate Peripheral pattern of HIE}

Typically seen in neonates with mild to moderate insult, due to decreased perfusion to the parasagittal cortical and subcortical regions with redirection of the blood flow to more metabolically active regions of the brain, such as the thalami, basal ganglia, brainstem and cerebellum (15). Prolonged events result in injury to the intervascular border (watershed) areas in these regions (15). Ultrasound should evaluate the grey-white matter differentiation, to look for areas of abnormal differentiation that could be abnormally accentuated, decreased or show a mixed pattern in the parasagittal frontal and parietal regions (13). Cortical thickness and echogenicity in the cortex and parasagittal frontalparietal cortex should also be carefully evaluated, with thickening and increased echogenicity seen in acute events (Figure 19) (14). Chronically, atrophic changes, volume loss and hypoechogenicities involving the subcortical white matter and cortex can be seen in the affected areas.

\section{Central pattern of HIE}

The central pattern of HIE appears with profound prolonged asphyxia, when there is acute decreased delivery of oxygen and blood to the brain (15). Regions of the brain with higher metabolism in the neonatal period, such as the thalami, basal ganglia and brainstem, are susceptible to these changes and the ones more affected.

The most typical pattern of severe prolonged hypoxia in the neonatal brain are areas of increased echogenicity in the basal ganglia and thalamus, particularly in the globus pallidus and ventral lateral nuclei of the thalamus (Figure 20) (14). In severe cases, they can cavitate with major neurological sequela. Foci of high echogenicity in the brainstem are less frequent and can be seen in severe cases and maybe difficult to see with CUS. Diffuse severe insults can affect also the cortex and subcortical white matter causing 

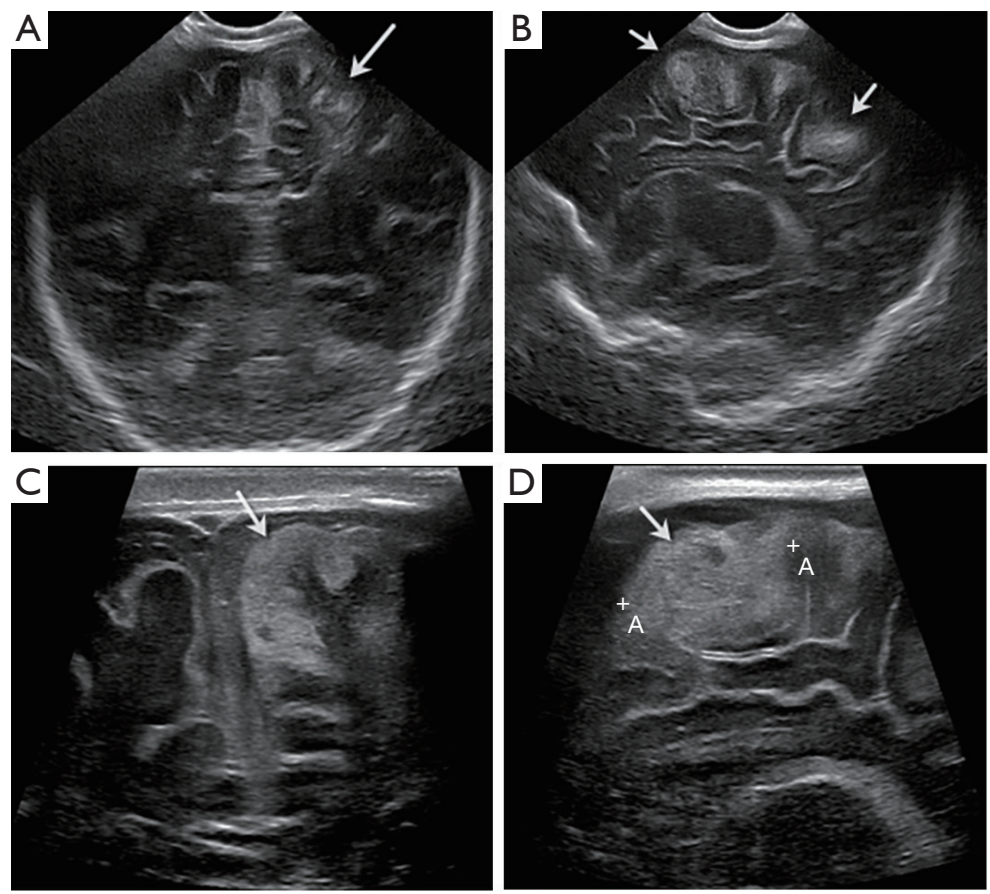

Figure 19 Term baby with perinatal asphyxia. Coronal (A) and sagittal (B) ultrasound images obtained with a curvilinear 11-MHz probe show a focus of abnormal echogenicity of the cortex and subcortical white matter (arrows) in the left precentral and postcentral cortex. Coronal (C) and sagittal (D) ultrasound images obtained with a linear 14-MHz transducer demonstrate increase echogenicity and swelling of the cortex, with abnormally increased and ill-defined white matter-grey matter differentiation $(+\mathrm{A})$.
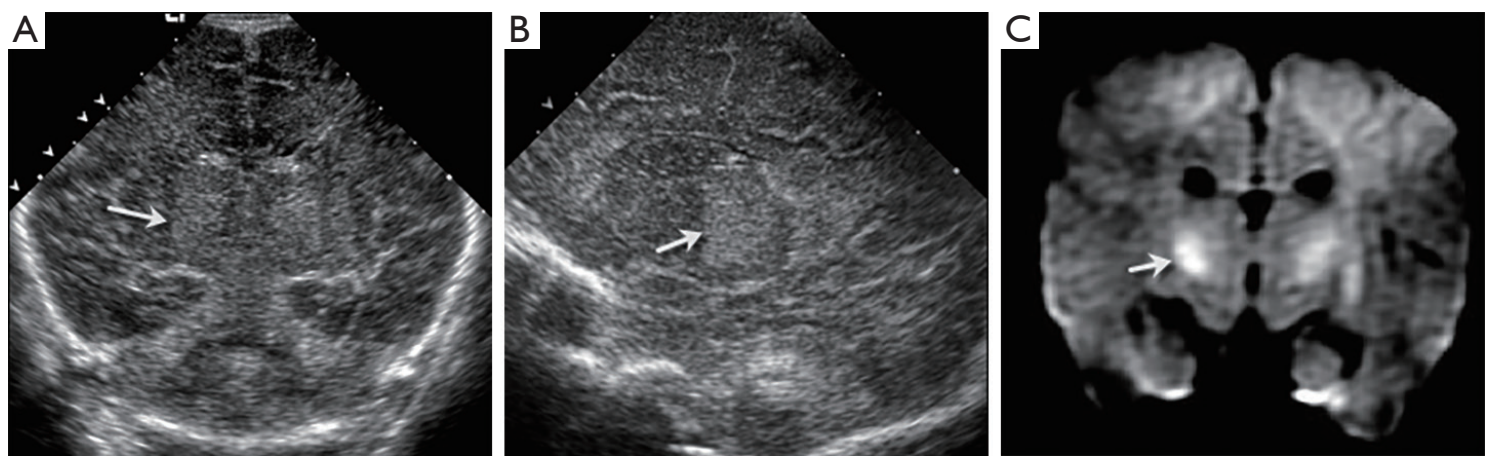

Figure 20 Fullterm neonate with a history of profound hypoxic-ischemic injury. Coronal (A) and sagittal (B) ultrasound images shows diffuse increased echogenicity of the basal ganglia (arrows) and slitlike ventricles. Coronal diffusion-weighted-imaging (C) shows restricted diffusion in the bilateral posterior putamen, corticospinal tracts and ventrolateral thalami (arrow).

generalized effacement of the sulci, loss of grey-white matter differentiation, and compressed/slit-like lateral ventricles (14).

\section{Neonatal arterial infarction}

Focal cerebral infarction in the neonate is uncommon, but more frequently seen in the term baby (16). It can be almost asymptomatic and diagnosed later in childhood when these children present with focal seizures of the contralateral limb. The middle cerebral artery distribution is the most frequent site. Initial CUS shows a loss of grey-white matter differentiation and mild mass effect (Figure 21). Doppler ultrasound is interesting as it can demonstrate abnormal perfusion in the middle cerebral arteries territories or even a thrombus. After a few weeks, volume loss and 

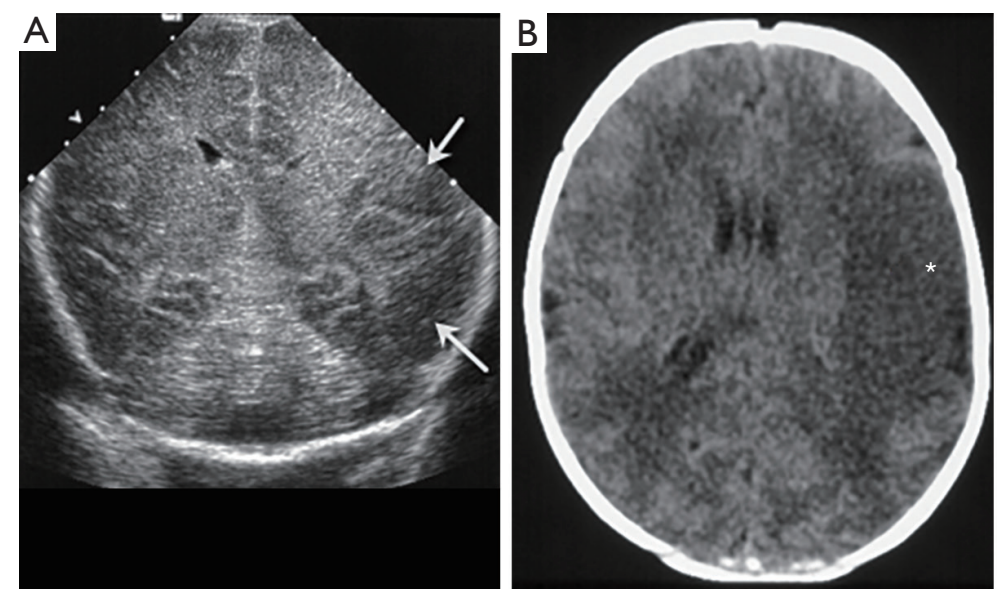

Figure 21 Coronal ultrasound image (A) obtained with an 11-MHz probe shows swelling and decreased echogenicity of the left perisilvian cortex (arrows) with loss of grey-white matter differentiation with mild mass effect over the left frontal horn. Axial unenhanced computed tomography (CT) image (B) confirms a hypodense focus (asterisk) in the region of the left middle cerebral artery, secondary to ischemia.
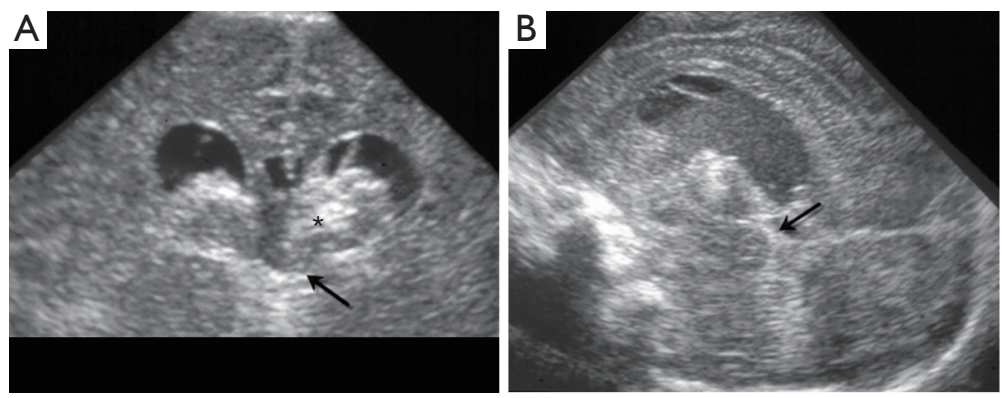

Figure 22 Coronal (A) and sagittal (B) ultrasound images obtained with 14-MHz linear and 11-MHz probes respectively demonstrate bilateral intraventricular haemorrhage (IVH) grade 3 (asterisk) that occupy and obstruct the foramina of Monro and the aqueduct (arrows) causing hydrocephalus.

cystic encephalomalacia become apparent. Haemorrhagic transformation of an ischemic stroke is rare in neonates.

\section{Cerebro-venous sinus thrombosis}

It may be a result of trauma, increased haematocrit, sepsis, dehydration, cardiac failure, and thrombotic disorders (4). The impaired venous drainage may cause venous infarction that can occur anywhere in the brain, the most characteristic locations are the thalami, resulting from thrombosis of the straight sinus and vein of Galen, and the bilateral parasagittal cortex and subcortical white matter, resulting from thrombosis of the superior sagittal sinus (4). Diffuse cerebral swelling with slit-like ventricles may be a result of extensive thrombosis. This diagnosis may be achieved with ultrasound by direct visualization of the thrombus or the lack of normal venous flow in the venous sinuses on Doppler ultrasound (6). Serial
Doppler ultrasound is an easily performed and inexpensive alternative for monitoring sinovenous thrombosis.

\section{Hydrocephalus}

CUS is really useful for the evaluation of children with macrocephaly, rapid increase in head circumference or a bulging fontanelle. It helps to quantify, monitor and confirm ventricular dilatation and to look for the cause (3). Hydrocephalus can be due to obstruction to the CSF outflow, decreased CSF absorption or CSF overproduction. The entire ventricular system should be evaluated to identify the cause of the obstruction and the level of transition from a large to a small ventricle (Figure 22). Dilation of the lateral and third ventricles point to an obstruction at the Sylvian aqueduct. Dilation 


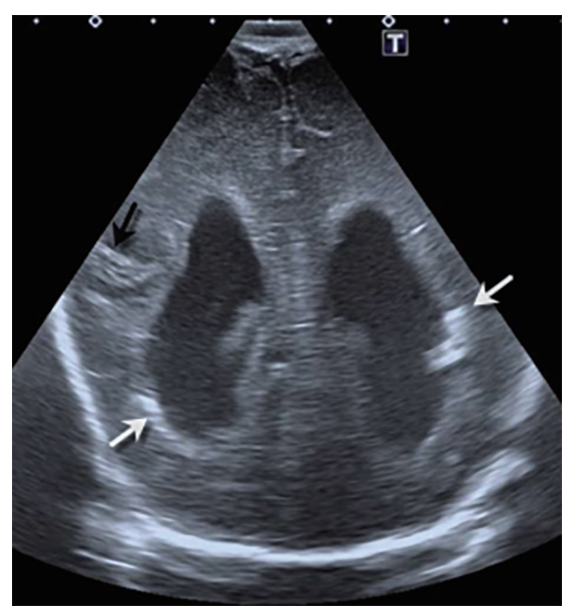

Figure 23 Newborn with cytomegalovirus (CMV) intrauterine infection. Coronal ultrasound image obtained with a curvilinear $11-\mathrm{MHz}$ probe demonstrates foci of high echogenicity in keeping with calcification (withe arrows), significant ventricular dilatation and cortical malformation in right Sylvian fissure (black arrow).

of all ventricles points to an extraventricular source, but ventricular enlargement does not always mean obstruction. Severe cases of hypoxic-ischemic injury result in large ventricles due to brain atrophy weeks after the insult rather than obstructive hydrocephalus (3). CUS can be used to monitor the degree of ventricular dilation, guide timing for potential management and to assess position of the intracranial tip of a ventriculo-peritoneal shunt.

\section{Congenital and neonatal brain infections}

\section{Congenital infections}

The most frequent congenital infections are known with the acronym TORCH [toxoplasma gondii, others, such as syphilis, rubella, cytomegalovirus (CMV) and herpes simplex virus type 2] (17).

$\mathrm{CMV}$ is the most common congenital viral infection and the brain is the most commonly affected organ. It is associated with mental retardation, blindness, deafness and seizures (17). Depending on the time the infection during fetal development, children can have severe abnormalities (before 20 to 24 weeks) such as microcephaly, periventricular calcifications, lissencephaly, cerebellar hypoplasia, cortical dysplasias, porencephaly, and/ or multicystic encephalomalacia (Figure 23) (17). After 24 weeks, the neurological damage will be less severe.

Toxoplasmosis is the second most common congenital

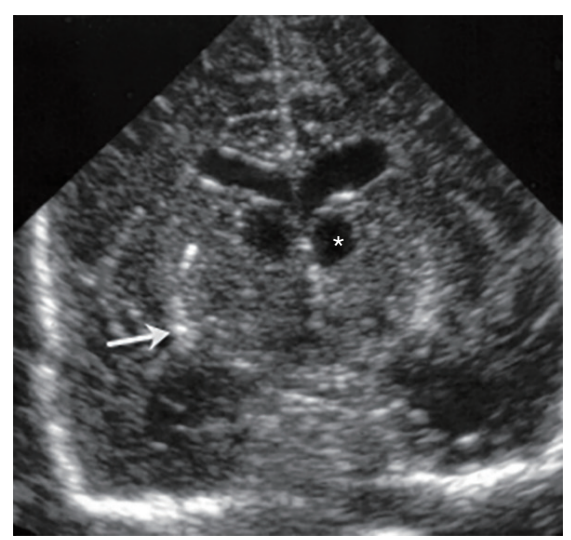

Figure 24 Newborn with toxoplasmosis intrauterine infection. Coronal ultrasound image obtained with a curvilinear $11-\mathrm{MHz}$ probe demonstrates bilateral symmetrical subependymal cysts (asterisk) and mineralizing vasculopathy (arrow).

infection. Similar to CMV, early in utero infection causes more severe cerebral anomalies, such as intrauterine growth restriction, hydrocephalus, microcephaly, calcifications, porencephaly or hydranencephaly (17). After 20 weeks, the sequelae are less severe but still include blindness, epilepsy and mental retardation (17). Non-shadowing cerebral and hepatic calcifications are the most typical ultrasound findings. Intracranial calcifications may be periventricular or random in distribution. Other less specific imaging findings are subependymal cysts, LSV and cystic white matter changes (Figure 24) (17).

\section{Neonatal acquired infections}

Neonatal brain infections are an important cause of severe long-term neurologic sequelae. The most common bacterial infections are Escherichia coli, group B Streptococci, Streptococcus pneumoniae and Haemophilus influenza (18). Imaging is needed only to evaluate for complications, such as subdural empyemas, cerebritis, abscess, ventriculitis and venous sinus thrombosis. Infarctions can occur from arterial vasculitis or venous sinus thrombosis (Figure 25). Ventriculitis appears on sonography as hydrocephalus, echogenic debris within the ventricles, septa within the ventricles and increased echogenicity.

Herpes type 2 can cause foetal and neonatal cerebral infections (18). It is a serious risk for children of mothers who have their first outbreak of this infection during pregnancy. It can be transmitted prenatally or at birth. In utero infection resembles TORCH infection, while postnatal infection involves the bilateral grey and white 

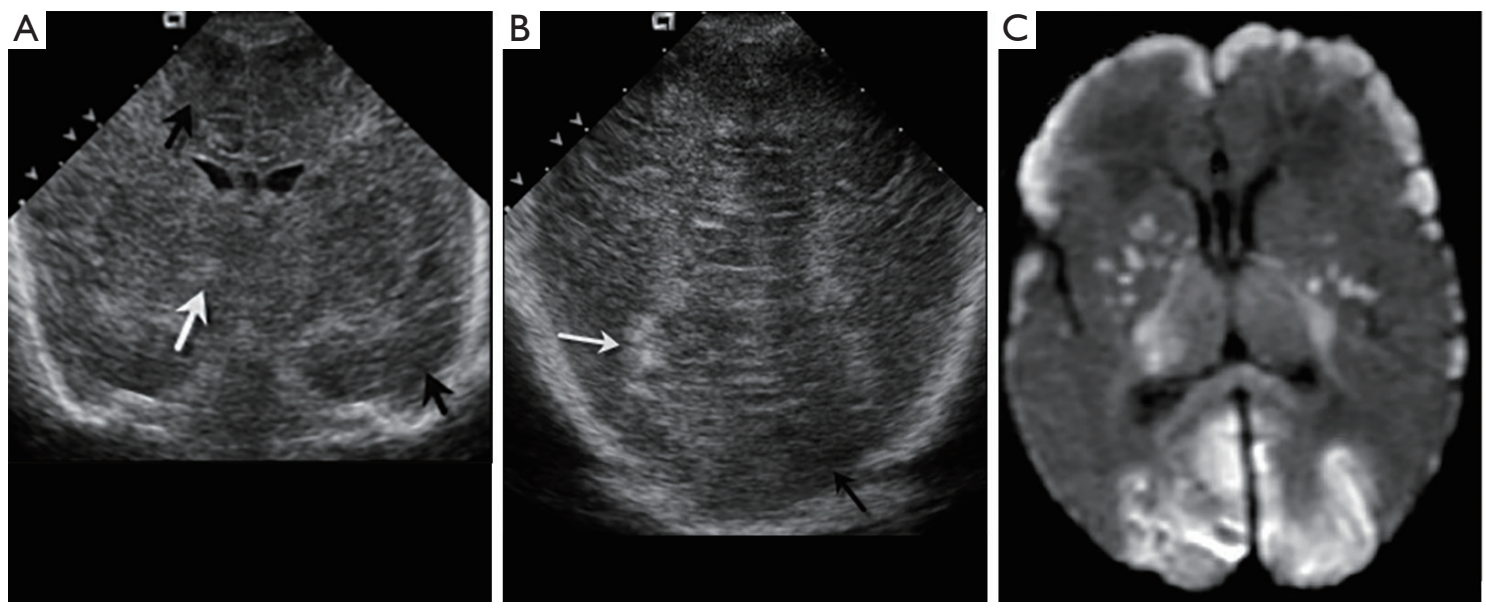

Figure 25 Term baby with group B streptococus encephalitis during the neonatal period. Coronal ultrasound images at the level of the frontal horns of the lateral ventricles (A) and the occipital lobes (B) show foci of abnormal high echogenicity in the white matter (black arrows) and right basal ganglia (white arrow) and foci of low echogenicity in the frontal and temporal cortex (black arrows). Axial diffusion-weighted magnetic resonance imaging (MRI) (C) confirmed the presence of areas of post-infectious acute infarction in the basal ganglia bilaterally and in the cortex of both frontal, temporal and occipital lobes.
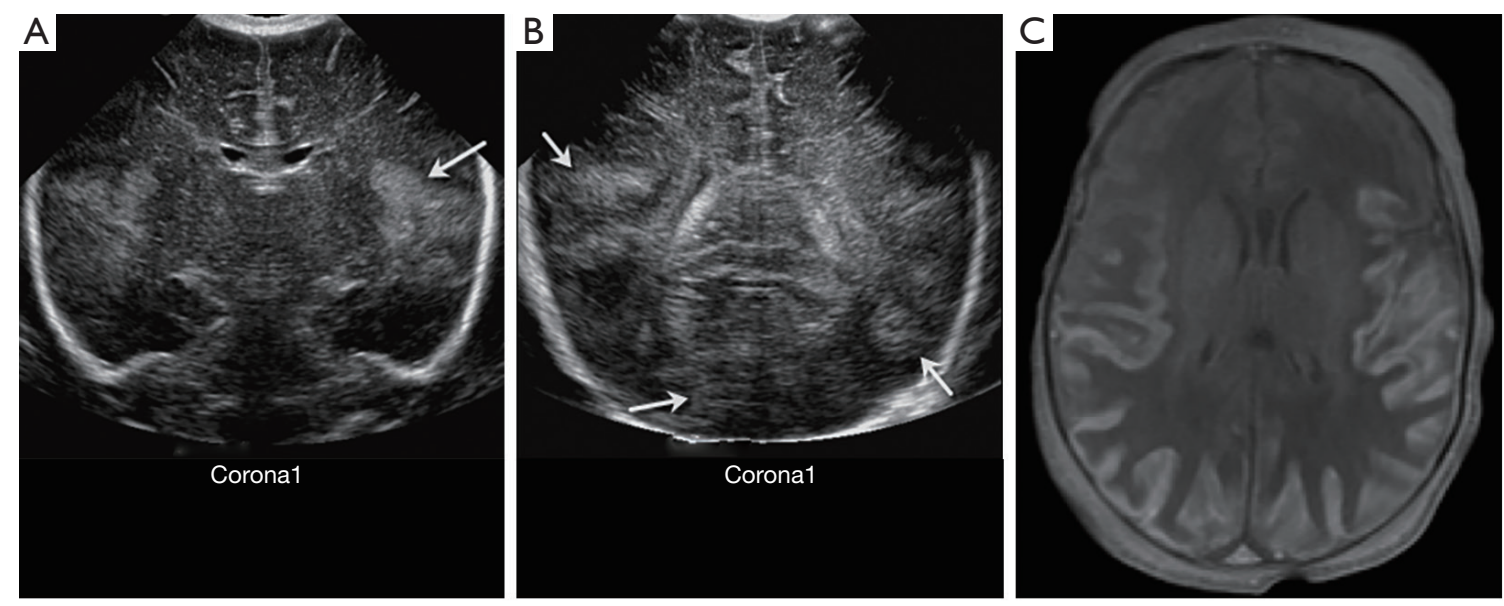

Figure 26 Neonate with herpes simplex virus encephalitis. Initial coronal ultrasound images through the frontal (A) and occipital (B) lobes show mild accentuation of the grey-white matter differentiation with diffuse blurring of the sylvian fissures [arrow in (A)] and abnormal thickness and echogenicity of the parieto-occipital cortex [arrows in (B)]. Axial non-contrast T1 magnetic resonance image (C) showed laminar hyperintensities in the bilateral parieto-occipital cortex consistent with cortical laminar necrosis.

matter diffusely, which may rapidly turn into whole brain necrosis. Multiple cortical haemorrhagic foci and sometimes leptomeningeal enhancement often are present (Figure 26). With time, cystic white matter changes develop.

\section{Solid and cystic masses}

Solid brain tumours during the first year of life are rare.
Some of them, might present with macrocephaly and CUS will be performed as a screening tool. Ultrasound can delineate the tumour site and size and evaluate cystic or solid components (19). Magnetic resonance imaging (MRI) will be the tool to characterize and delineate the tumour precisely. The most frequent ones are astrocytoma, medulloblastoma, ependymoma, atypical teratoid rhabdoid tumour, teratoma, embryonal tumour with multilayered rosettes (Figure 27) and 

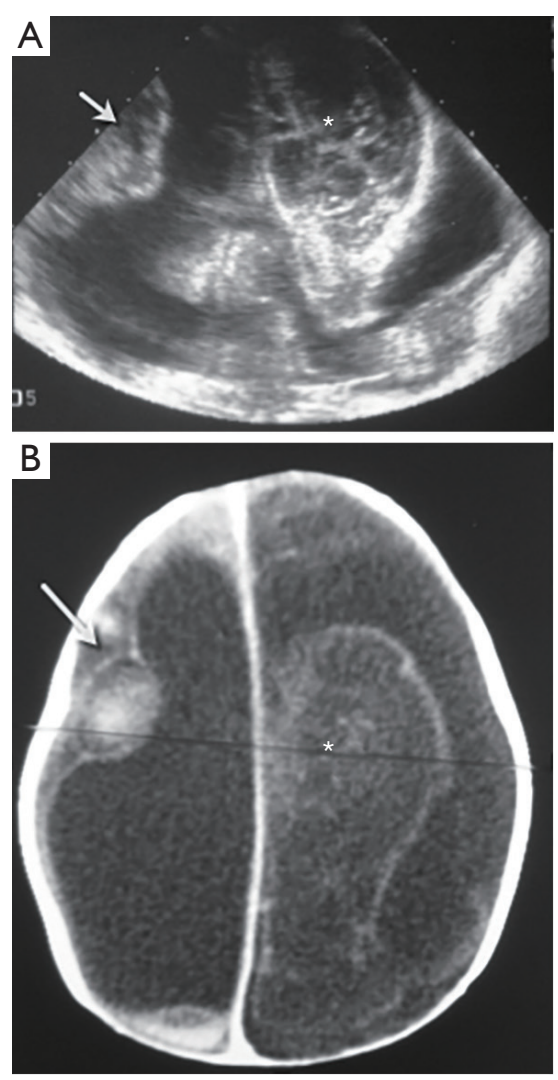

Figure 27 Newborn with embryonal tumour with multilayered rosettes. Coronal ultrasound image (A) obtained with an $11-\mathrm{MHz}$ ultrasound probe demonstrates a large solid heterogenous mass in the left ventricle (asterisk) and a smaller solid mass (arrow) in the right cerebral hemisphere with generalized ventricular dilatation. Axial contrast-enhanced computed tomography (CT) image (B) confirmed the findings.

craniopharyngioma. The ultrasound appearances of brain tumours are variable and nonspecific (19).

Arachnoid cysts are not rare in children, especially in the middle cranial and posterior fossae (3). Those located in the midline, may cause obstruction of the ventricular system, present with hydrocephalus and are frequently associated with other brain anomalies (Figure 28).

\section{Brain malformations}

Most brain malformations are diagnosed prenatally by ultrasound and MRI is the gold standard for postnatal characterization. Neonatal ultrasound can help to define and monitor them (2).

\section{Holoprosencephaly}
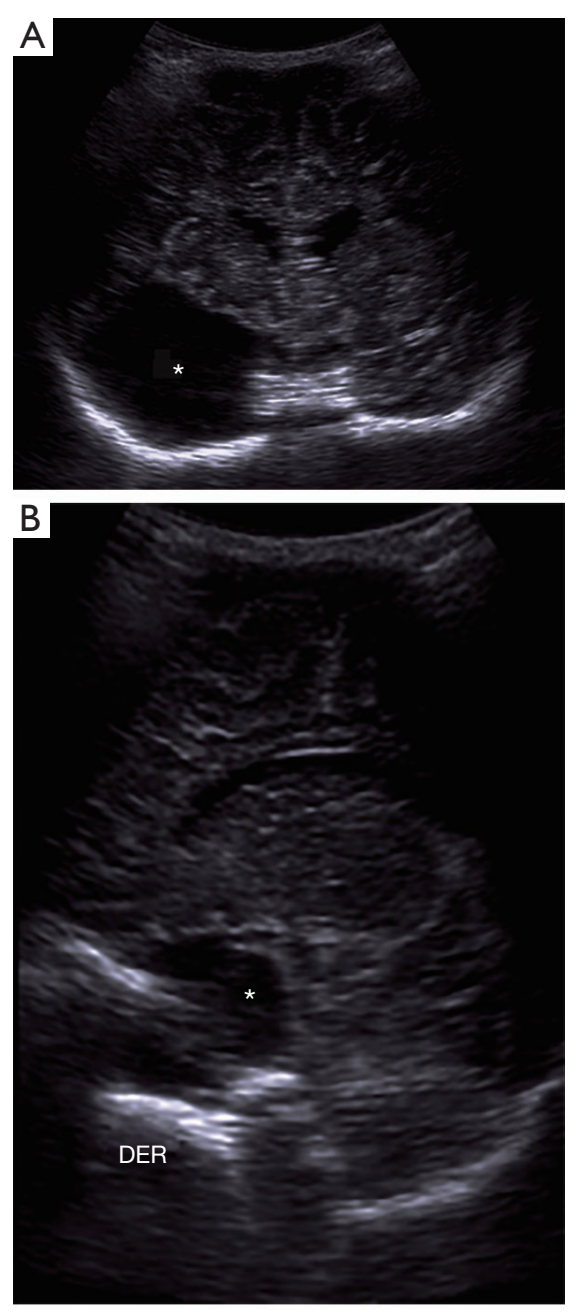

Figure 28 Term baby with arachnoid cyst. Coronal (A) and sagittal (B) ultrasound images obtained with an $11-\mathrm{MHz}$ probe show a large cystic lesion in the right middle cranial fossa with mild mass effect over the surrounding brain parenchyma, in keeping with an arachnoid cyst (asterisks).

Spectrum of malformations caused by a diverticulation failure of the primitive prosencephalon that does not divide into the telencephalon and the diencephalon (3). It varies from most severe, alobar holoprosencephaly, with no separation of the brain hemispheres (Figure 29), to least severe with partial separation of the posterior aspects of the brain (lobar holoprosencephaly). The septum pellucidum is always absent.

\section{Grey matter heterotopia}

Grey matter heterotopia is a cortical malformation secondary to the interruption of normal neuronal migration from the surface of the ventricle to the cortex. The nodular 


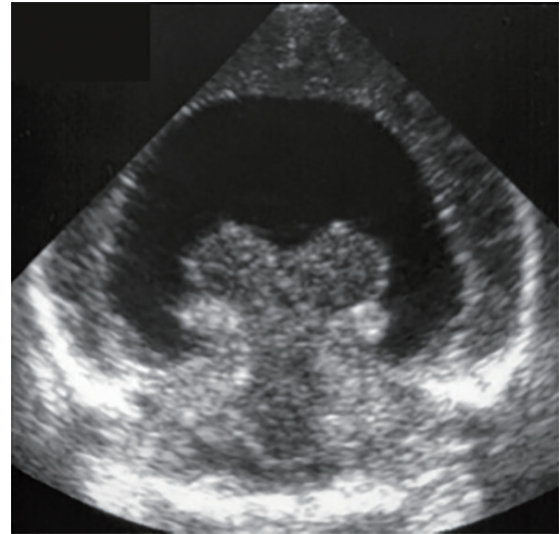

Figure 29 Newborn with holoprosencephaly. Coronal ultrasound image obtained with an 11-MHz probe shows absence of separation of the brain hemisphere and thalami, with absence of septum pellucidum. form can mimick a "pseudomass" appearance with abnormal adjacent cortex (Figure 30).

\section{Galenic venous malformation}

Dilation of the vein of Galen (incorrectly called aneurysm, better called median prosencephalic arteriovenous fistula) present in early childhood with features of left-to-right shunt and high output cardiac failure (3). On ultrasound, it appears as a midline anechoic cystic mass posteriorly, with prominent blood flow on Doppler (Figure 31). Endovascular embolization is the treatment of choice.

\section{Take home messages}

(I) Ultrasound is the initial screening tool for infants and
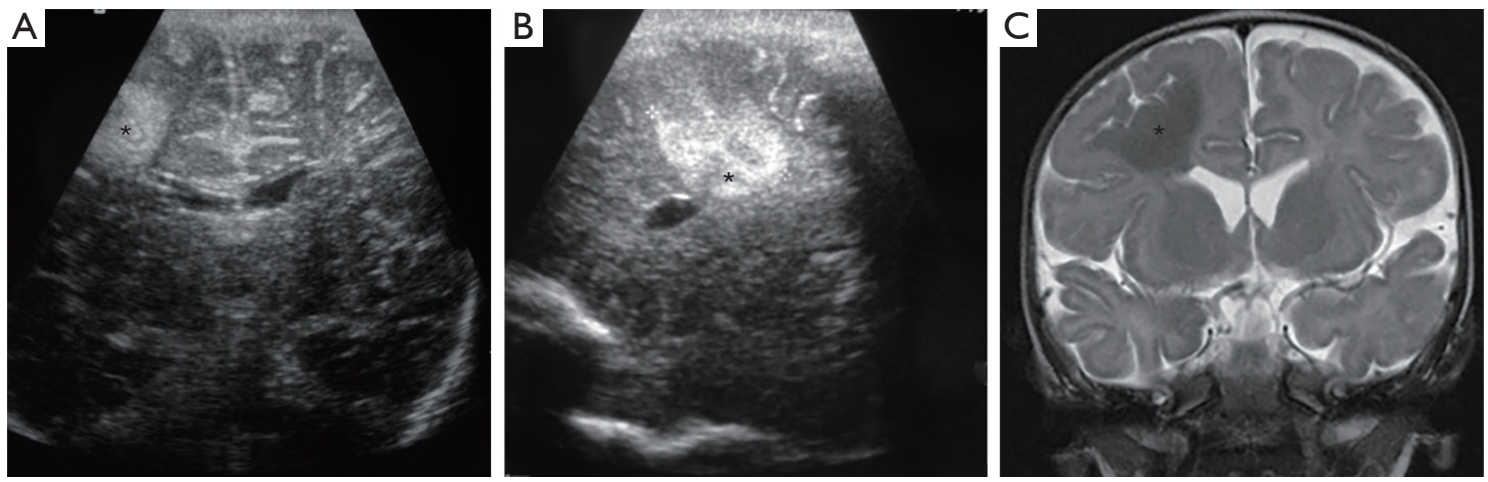

Figure 30 Newborn with focal nodular heterotopia. Coronal (A) and sagittal (B) ultrasound images obtained with a curvilinear ultrasound probe demonstrate a large hyperechoic lesion in the right frontal region (asterisks), with abnormal overlying cortex. Coronal magnetic resonance $\mathrm{T} 2$ image $(\mathrm{C})$ confirmed the findings.

complementary to computed tomography (CT) and MRI.

(II) Use a systematic technique and standardized report, always checking that you have visualized and documented adequately all relevant anatomical structures.

(III) Use the anterior and mastoid fontanelles and two types of transducers, the curvilinear for deeper structures, and the high-frequency linear for superficial ones.

(IV) Be familiar with the most common ultrasound anatomical variants.

(V) In premature babies look for IVH and PVL.

(VI) In term babies look for the central pattern of hypoxia in the basal ganglia and thalami and the peripheral pattern in the watershed areas.

\section{Conclusions}

CUS is an accessible, useful and harmless technique that can be used bedside repeatedly during the first year of life. This article has explained indications, basic technical parameters, scanning technique, standardised planes, common variants and the most frequent abnormal findings, with special emphasis on intracranial haemorrhage and hypoxic-ischemic events of the premature and term baby.

\section{Acknowledgments}

We would like to thank Dr. Alan Daneman from the Hospital for Sick Children, Toronto, for his contribution 

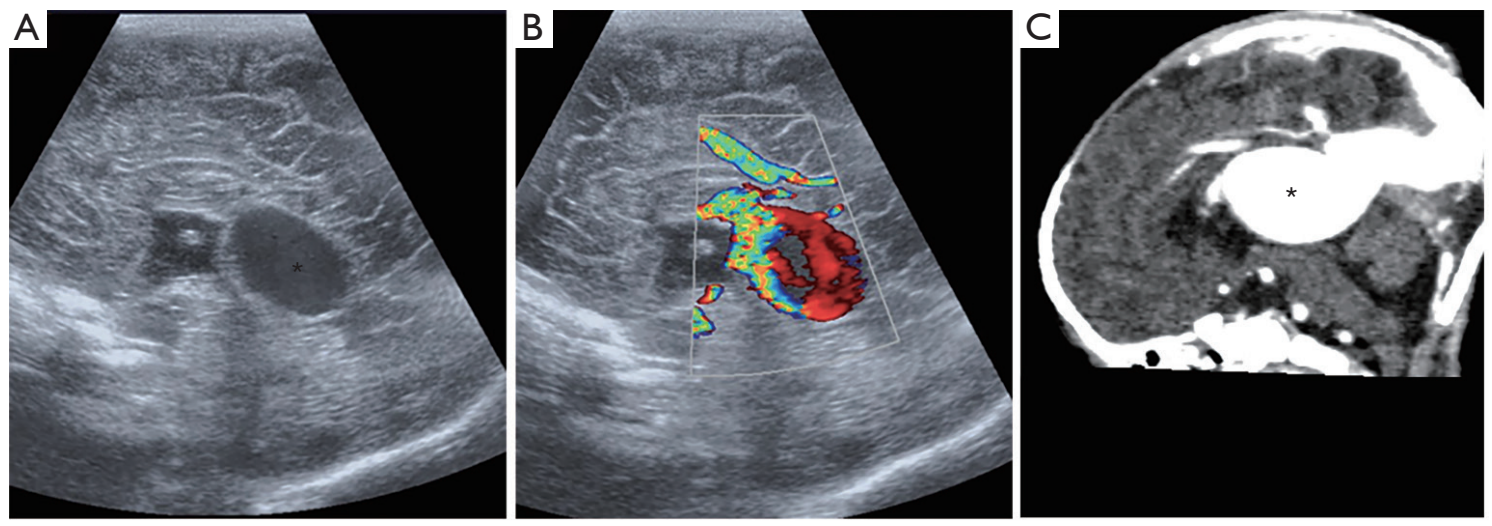

Figure 31 Term baby with vein of Galen malformation. Sagittal ultrasound (A), colour Doppler (B) and contrast-enhanced computed tomography (CT) (C) images demonstrate a posterior midline cystic lesion (asterisk) with marked blood flow on colour Doppler, in keeping with dilatation of the median prosencephalic vein.

with images to this article and his passionate teaching on this field.

Funding: None.

\section{Footnote}

Provenance and Peer Review: This article was commissioned by the Guest Editor (Felice D'Arco) for the series "Pediatric Neuroradiology for Trainees and Fellows: An Updated Practical Guide" published in Translational Pediatrics. The article has undergone external peer review.

Peer Review File: Available at http://dx.doi.org/10.21037/tp20-399

Conflicts of Interest: All authors have completed the ICMJE uniform disclosure form (available at http:// dx.doi.org/10.21037/tp-20-399). The series "Pediatric Neuroradiology for Trainees and Fellows: An Updated Practical Guide" was commissioned by the editorial office without any funding or sponsorship. The authors have no other conflicts of interest to declare.

Ethical Statement: The authors are accountable for all aspects of the work in ensuring that questions related to the accuracy or integrity of any part of the work are appropriately investigated and resolved.

Open Access Statement: This is an Open Access article distributed in accordance with the Creative Commons Attribution-NonCommercial-NoDerivs 4.0 International
License (CC BY-NC-ND 4.0), which permits the noncommercial replication and distribution of the article with the strict proviso that no changes or edits are made and the original work is properly cited (including links to both the formal publication through the relevant DOI and the license). See: https://creativecommons.org/licenses/by-nc-nd/4.0/.

\section{References}

1. AIUM practice parameter for the performance of neurosonography in neonates and infants. J Ultrasound Med 2020;39:E57-61.

2. Siegel M. Brain. In: Siegel MJ. editor. Pediatric sonography. 4th ed. Philadelphia: Lippincott Williams \& Wilkins, 2011:43-117.

3. de Bruyn R. Pediatric ultrasound: how, why and when. 1st ed. Philadelphia: Elsevier, 2005.

4. Wolf R. Transfontanellar ultrasound in preterm infantsa survival guide for the young radiologist on call. Vienna: European Congress of Radiology, 2017. doi: 10.1594/ ecr2017/C-1668.

5. Steggerda SJ, de Bruïne FT, Smits-Wintjens VE, et al. Ultrasound detection of posterior fossa abnormalities in full-term neonates. Early Hum Dev 2012;88:233-9.

6. Raets MM, Sol JJ, Govaert P, et al. Serial cranial US for detection of cerebral sinovenous thrombosis in preterm infants. Radiology 2013;269:879-86.

7. Maller VV, Cohen HL. Neurosonography: assessing the premature infant. Pediatr Radiol 2017;47:1031-45.

8. Fabre C, Tosello B, Pipon E, et al. Hyperechogenicity of lenticulostriate vessels: A poor prognosis or a normal 
variant? A seven year retrospective study. Pediatr Neonatol 2018;59:553-60.

9. Epelman M, Daneman A, Blaser SI, et al. Differential diagnosis of intracranial cystic lesions at head US: correlation with CT and MR imaging. Radiographics 2006;26:173-96.

10. Hosseinzadeh K, Luo J, Borhani A, et al. Non-visualisation of cavum septi pellucidi: implication in prenatal diagnosis? Insights Imaging 2013;4:357-67.

11. Hand IL, Shellhaas RA, Milla SS, et al. Routine neuroimaging of the preterm brain. Pediatrics 2020;146:e2020029082.

12. Limperopoulos C, Benson CB, Bassan H, et al. Cerebellar hemorrhage in the preterm infant: ultrasonographic findings and risk factors. Pediatrics 2005;116:717-24.

13. Parodi A, Rossi A, Severino M, et al. Accuracy of ultrasound in assessing cerebellar haemorrhages in very low birthweight babies. Arch Dis Child Fetal Neonatal Ed

Cite this article as: Caro-Domínguez P, Lecacheux C, Hernandez-Herrera C, Llorens-Salvador R. Cranial ultrasound for beginners. Transl Pediatr 2021;10(4):1117-1137. doi: $10.21037 /$ tp-20-399
2015;100:F289-92.

14. Epelman M, Daneman A, Kellenberger CJ, et al. Neonatal encephalopathy: a prospective comparison of head US and MRI. Pediatr Radiol 2010;40:1640-50.

15. Chao CP, Zaleski CG, Patton AC. Neonatal hypoxicischemic encephalopathy: multimodality imaging findings. Radiographics 2006;26 Suppl 1:S159-72.

16. Deeg KH. Sonographic and Doppler sonographic diagnosis of neonatal ischemic stroke. Ultraschall Med 2017;38:360-76.

17. Neuberger I, Garcia J, Meyers ML, et al. Imaging of congenital central nervous system infections. Pediatr Radiol 2018;48:513-23.

18. Schneider JF. Neonatal brain infections. Pediatr Radiol 2011;41 Suppl 1:S143-8.

19. Simanovsky N, Taylor GA. Sonography of brain tumors in infants and young children. Pediatr Radiol 2001;31:392-8. 